\title{
Semantic memory: Complexity or connectivity?
}

\author{
NEAL E. A. KROLL \\ University of California, Davis, California \\ and \\ WOLFGANG KLIMESCH \\ University of Salzburg, Salzburg, Austria
}

\begin{abstract}
The complexity hypothesis predicts that concept-feature verification times should be directly related to the number of features associated with a concept (the fan effect). Conversely, the connectivity hypothesis predicts that for interconnected semantic relationships, this verification time should be inversely related to the number of closely associated features (the reversed fan effect). In the present experiments, although the time required for episodic recognition memory decisions tended to be directly related to the number of features associated with a concept, semantic verification times were inversely related to the complexity of the concept. In addition, the concept's complexity was at least as good a predictor of performance in semantic tasks as was the strength of association between the concept and feature. Both of these results are interpreted in terms of the connectivity hypothesis.
\end{abstract}

Many models of semantic memory are based on the assumption that the meanings of concepts can be represented by sets or networks of semantic features or properties (see, e.g., Chang, 1986; Collins \& Loftus, 1975; Collins \& Quillian, 1970; Klimesch, 1981; McNamara \& Sternberg, 1983; Smith \& Medin, 1981; Smith, Shoben, \& Rips, 1974). Crucial to this assumption is that the meaning of a concept can be decomposed into its semantic elements, which in turn raises the question of the way in which the complexity of a concept (i.e., its number of features) influences memory load and processing time. The usual answer to this question is based on the hypothesis that the processing capacity for accessing and comparing features is limited, with the consequence that more processing time is required as more semantic features need to be accessed and compared (see, e.g., Smith \& Medin, 1981, p. 43 ff.). For our present purposes, this answer, regardless of the precise model that generates it, will be referred to as the complexity hypothesis (see Gentner, 1981).

The apparent naturalness of the preceding answer has led to such a close linking of the complexity hypothesis and the assumption of decomposition that results confirming the complexity hypothesis have been viewed as also confirming the assumption of decomposition, and vice versa. Thus, after reviewing a series of experiments that do not support the complexity hypothesis, Kintsch (1974, 1980) was inclined to conclude that concepts are not represented by features, but rather by larger "supraword" units. However, as Gentner (1981) has pointed out, there is

This research was supported in part by a Grant from the Max Kade Foundation to the second author. Requests for reprints should be sent to Neal Kroll, Department of Psychology, University of California, Davis, CA 95616-8686. another possibility. That is, perhaps the decomposition assumption is correct, but the complexity hypothesis is not.

An alternative view of the role played by a concept's features has been labeled the connectivity hypothesis (Gentner, 1981). The connectivity hypothesis states that the greater the number of connections among semantic components, the greater the enhancement of memory performance and the faster the retrieval. Until recently, however, experimenters testing the relative merits of the complexity and connectivity hypotheses (e.g., Anderson, 1981; Anderson \& Reder, 1987; Gentner, 1981; Myers, O’Brien, Balota, \& Toyofuku, 1984; Reder \& Ross, 1983) have measured the retrieval of recently learned facts rather than the processing of natural concepts, which are the main consideration of semantic memory models (see Chang, 1986, for a recent review of semantic memory theories and related experiments).

Klimesch (1987, 1988, in press) has proposed a version of the connectivity model for semantic processing. This model is based on four major assumptions: one about representation, and three about processing.

The assumption about representation is that semantically related informational units, such as natural concepts, are not represented well by independent fan-like, hierarchical structures. Rather, natural concepts contain features that are semantically related to each other and, hence, are better represented by an interconnected structure of features.

The first assumption about processing is that activation occurs not only directly, as suggested by the models with fan-like structures, but also indirectly. Thus, as the number of features increases, so does the number of interrelating pathways, and, accordingly, the greater will be the amount of indirect activation that can spread back to the concept node, thereby speeding the processing time. Activation leaving a node on any given pathway is as- 
sumed to be relatively unaffected by the number of pathways connected to that node ${ }^{1}$ - unlike in the typical complexity model, in which the amount of activation leaving a node is fixed so that the amount of activation leaving on any given pathway would be an inverse function of the number of such pathways.

The second assumption about processing is that a search process in semantic memory terminates when indirect activation spreads back to the node from which the activation process originated. The time that indirect activation needs to spread back is termed $t(\mathrm{I})$. This cycling back of the activation signals positive evidence of a successful search. The usual evidence of an unsuccessful search, however, is the lack of such a return of activation. Thus, a corollary of the second assumption is that the search process also terminates if no indirect activation is received after the critical time $t(\mathrm{c})$ has elapsed.

The third assumption about processing is that semantic processing time is a function of the amount of indirect activation $I$. Since $I$ reflects the amount of positive evidence, semantic processing time and the amount of indirect activation vary inversely: the more positive evidence $I$ that a search process yields, the shorter will be the processing time. It is important to note that it is $I$, via an accumulator process, and not $t(\mathrm{I})$ per se, that is assumed to be the crucial predictor for a subject's reaction time. (Ratcliff \& McKoon, 1981, have shown that the time of spreading activation is so fast that it can hardly be considered a good predictor of the results of reaction time experiments.)

A more complete description of Klimesch's (1987, 1988, in press) connectivity model is presented in Appendixes A and B. For the present purposes, however, the most important prediction of the connectivity model is that concepts with many features should be processed faster than concepts with only a few features. ${ }^{2}$

In order to test his model, Klimesch (1987, Experiment 1) first determined the number of semantic features for a sample of concepts and, at the same time, provided evidence for two very basic propositions from his connectivity model: first, that subordinate concepts $X$ do indeed share common features with their superordinate categories $Y$; and second, that the number of common features, shared by $X$ and $Y$, is significantly correlated with typicality, as defined by Rosch (1975).

Klimesch (1987) then provided two additional experiments in which subjects performed a semantic judgment task of the type "Does concept $X$ belong to superordinate category $Y$ ?" In these experiments, Klimesch tested five hypotheses that were derived from his connectivity model.

1. For positive decisions, the greater the number of features in the concept $X$, the faster the verification time (VT).

2. For positive but not negative decisions, the more common features shared by concepts $X$ and $Y$, the faster the VT.

3. For positive but not negative decisions, the greater the number of features in superordinate category $Y$, the faster the VT.
4. Negative decisions will also be faster as a function of the number of features in concept $X$, but to a much smaller extent than will positive decisions.

5. Negative VTs will be longer on the average than positive VTs.

The results of Klimesch's (1987) three experiments provided evidence in favor of the two basic propositions and each of the five hypotheses.

In the present series of experiments, Experiments $2 \mathrm{~A}$ and 4 change the semantic judgment task and test an additional hypothesis generated by the connectivity model. The task is now to judge the congruency between a natural concept and a potential feature of that concept, with half of the subjects seeing the concept word first and half seeing the feature word first. The assumption is that decisions in this congruency task are made in the same way as that used in the semantic judgment task described above. In this congruency task, a positive decision indicates that the feature word $Y$ (e.g., YELLOW) represents a property, feature, or attribute of the paired concept $X$ (e.g., Canary). The connectivity model assumes that the amount of indirect activation spreading back and accumulating at the concept node $X$ is a factor affecting VT. Thus, the amount of indirect activation is a function of the number of features belonging to concept $X$. The more the features belonging to concept $X$, the more the indirect activation will spread back to $X$ and $Y$.

One important issue remains to be discussed. What does it mean, in terms of the connectivity model, to detect that $Y$ is a feature of $X$ ? To answer this question, we refer to additional assumptions of the connectivity model (Klimesch, 1987, p. $55 \mathrm{ff}$.). The following argument is based on the situation in which the order of presentation consists of feature to concept $(Y \rightarrow X)$.

Let $I(x)$ be the amount of indirect activation that would occur from presenting only $X$, and assume that $I(x)$ is stored with the code $X$. In contrast, let $I(y x)$ be the amount of indirect activation received via the activation of $Y$. The search criterion, then, refers to the comparison of $I$ with $I(x)$, where $I$ is the result of any actual search process. On congruent trials, where $Y$ is part of the feature network of $X$, activation will flow from $Y$ to $X$ so that $I$ will increase from $I(x)$ to $I(x)+I(y x)$. The relationship $I>I(x)$, then, indicates that $Y$ is part of $X$, resulting in a positive decision. If, however, $I=I(x), Y$ cannot have been a feature of $X$ and a negative decision results. In both cases, the complexity of $X$ speeds processing time.

Thus, for reasons similar to those advanced for the semantic judgment task used in earlier studies (Klimesch, 1981, 1987), concepts with many features should be retrieved and compared faster than those with only a few features-that is, a reversed fan effect. The connectivity model thus predicts that VT should be conversely related to complexity: The more features a natural concept comprises, the faster should be the congruency judgmentregardless of presentation order (i.e., concept-feature or feature-concept). ${ }^{3}$ In fact, it is to be expected that VTs 
in a semantic congruency task should be at least as much a function of the complexity of the concept as of the position of the feature in the concept's hierarchy of features.

It should be emphasized that the predictions described above will be valid only if the features of a concept do indeed form an interconnected structure. Let $\tilde{n}$ be the number of core features that the concept comprises. By definition, the concept node must be connected to all of the relevant feature nodes, so the number of connections between the concept node and its features is $\tilde{n}$ and the maximum possible number of interconnections among the $\tilde{n}$ features for an interconnected network is $n(n-1) / 2$. However, the model predicts the complexity effect above even if the number of interconnections is less than this maximum, if at least a minimum degree of interconnectivity is fulfilled. This minimum requirement is that each of a number of features is connected to at least one of the other features in such a way that a closed structure emerges. (See Appendix A for a more detailed description of the theory.)

Note that with increasing $\tilde{n}$, the maximum possible number of interconnections among the features increases exponentially. Because of this potentially exponential nature of the relationship between $\tilde{n}$ and the number of connections, we were initially unconcerned about a potential negative relationship between the number of features and the strength of connections among the concept and its features. We believed that to find a correlation between a measurement of the number of features and speed of decision making would be enough to support our hypothesis. However, the editor and one of the reviewers of an earlier version of this manuscript convinced us not only that a negative relationship between number of features and strength of connections was possible (and likely), but also that it might detract from our theory. That is, if the interconnections among the features of the concepts with larger $n$ values were either missing or even much weaker than those of concepts with smaller $\tilde{n}$ values, any correlations between $\tilde{n}$ and decision times would have to be due to something other than connectivity. Consequently, we measured the strengths of individual connections by asking subjects to directly rate the strength of each of the relevant concept-feature and feature-feature connections. Measuring the total of the average ratings of all the connections in the feature network of a concept is taken to reflect the degree of connectedness. Had this measure not correlated with the number of features, we would have had to assume that the degree of interconnectedness and the number of features are distinct measures. Our theory, however, led us to expect a close relationship: features of well-elaborated semantic structures (such as semantic structures reflecting well-known concepts) are not independent information units, but rather are highly interrelated. If this view is correct, a high correlation should be found between the connectedness ratings of concepts and the number of features associated with these concepts.

Although this experiment was, in fact, performed last, it is presented first so that measurements gained from the experiment may be referred to during the remainder of the report.

\section{EXPERIMENT 1}

\section{Method}

Material. Eight concept words were selected for each of six superordinate categories: BIRDS, CLOTHES, FRUITS, VEGETABLES, VEHICLES, and WEAPONS. Previous subjects had listed the features that they felt were most important in and characteristic of the description of these concepts (Klimesch, 1987). For example, one concept in the superordinate categogy VEGETABLES was CORN; characteristic features listed for CORN were YELLOW, COB, SWEET, HUSK, STALKS, and GOOD. The number-of-features (NOF) value represents the actual number of features listed by subjects. For CORN, the NOF value found from these subjects is 6 . A concept's number-of-attributes (NOA) value, on the other hand, represents the average rated number of attributes (or features) that subjects assign to that concept (Toglia \& Battig, 1978). For CoRN, the NOA value is 4.10. As would be expected, these two values tend to correlate highly with one another (Klimesch, 1987). For the concept words used in this experiment, the correlation between the NOA and NOF values was .530 . Note that, with the present model, it is not necessary to assume that either of these values measures the complete list of a concept's associated features. Rather, it is assumed that their relative magnitudes represent the relative magnitudes of the number of associated features that are highly integrated into the meaning of the concepts in a relatively contextfree situation. ${ }^{4}$

Procedure. The words were presented on a computer monitor, and the subjects rated the relationships via the computer keyboard. Each subject rated the relationships among the words from a single superordinate category. The fastest subject required approximately $45 \mathrm{~min}$, and the slowest required approximately $70 \mathrm{~min}$ to complete the rating task. The name of the superordinate category was always listed at the top of the monitor, and the subject was told to interpret the words in the context of this category. (Thus, for example, the superordinate category name WEAPONS informed the subject that the concept word CluB was a weapon and not a card suit.) The rating scale went from 0 (no relationship) to 6 (very strong relationship).

The subject first rated the connections between the concept and each of its features (previously listed by subjects in Klimesch, 1987). For example, if the superordinate category was VEGETABLES and the concept was CUCUMBER, the subject would rate the connection between CUCUMBER and each of its features, GREEN, SEEDS, and LONG. When all of the concept-feature ratings were completed for a given concept, they were listed on the screen in the order of their ratings, with the rating given beside the feature word. Thus the subject not only saw the rating that had been given to each, but also saw the relative rankings. The subject could then change any of the ratings in order to better reflect their relative standings.

After the subject had settled on the appropriate ratings for the concept-feature pairs, the subject was shown all possible pairings of these features and asked to rate the strength of relationship between them. For example, after rating CuCumber-Green, CuCumberSEEDS, and CUCUMBER-LONG, the subject rated SEEDS-GREEN, and SEEDS-LONG. Then the subject saw these words and ratings ranked in the order of the ratings and was allowed to change the ratings so that the proper rank order of relationship was represented. Then the subject saw all of the features again, starting with one of the other features-for example, LONG-and rated the relationships from this base: LONG-SEEDS, LONG-GREEN, until all possible combinations of features had been rated for a particular concept. (The subjects were told that the $X \rightarrow Y$ relationship was not necessarily the same as the $Y \rightarrow X$ relationship, but these rating relationships were, 
Table 1 Correlations Between Pairs of Measures

\begin{tabular}{lccclll}
\hline & NOA & NOF & $\Sigma C f$ & $\Sigma f f$ & Net & Average Cf \\
\hline NOF & $.530^{*}$ & & & & & \\
$\Sigma C f$ & $.606^{*}$ & $.942^{*}$ & & & & \\
$\Sigma$ ff & $.525^{*}$ & $.928^{*}$ & $.924^{*}$ & & & \\
Net & $.552^{*}$ & $.945^{*}$ & $.956^{*}$ & $.996^{*}$ & & \\
Average Cf & .208 & -.128 & .192 & .047 & .083 & \\
Average ff & $.500^{*}$ & $.647^{*}$ & $.737^{*}$ & $.761^{*}$ & $.766^{*}$ & .284 \\
\hline
\end{tabular}

Note-NOA, number of attributes (Toglia \& Battig, 1978); NOF, number of features (Klimesch, 1987). The following measures are for Experiment 1: $\Sigma \mathrm{Cf}$, total of average ratings of connection strength between concepts and their features; $\Sigma \mathrm{ff}$, total of average ratings of connection strength between pairs of features; Net, total of average ratings of all connections in the network (Net $=\Sigma \mathrm{Cf}+\Sigma \mathrm{ff}$ ). Average $\mathrm{Cf}=$ $\Sigma \mathrm{Cf} / \mathrm{NOF} . \quad$ Average ff $=2 \cdot \Sigma \mathrm{ff} /\left(\mathrm{NOF}^{2}+\mathrm{NOF}\right) . \quad{ }^{*} p<.002$

in practice, very similar and were consequently averaged during data summary.)

After the subject had rated all the feature pairs for a given concept, the next concept appeared and the subject rated the connections between this concept and each of its features, etc. The order of the concepts and the order of the features for each concept were varied across subjects.

Subjects. The subjects were 96 students in introductory psychology and rhetoric classes at the University of California, Davis, who received extra credit for participating in the experiment. The subjects all had English as their first language. Each superordinate category was rated by 16 subjects.

\section{Results}

The correlations between pairs of the main measures of interest are presented in Table 1. The measures NOA and NOF are discussed above and were found for these concepts previously by Toglia and Battig (1978) and Klimesch (1987), respectively. The total strength of the connections between a concept and its core features, $\Sigma \mathrm{Cf}$, was found for each concept by summing the average concept-to-feature ratings over all features for that concept. The total strength of the interconnections between the various pairs of features for each concept, $\Sigma$ ff, was found for each concept by summing the average featureto-feature ratings over all features for that concept. The net value for each concept represents the total of average ratings over all connections in the network; that is, net $=\Sigma \mathrm{Cf}+\Sigma \mathrm{ff}$. The average $\mathrm{Cf}$ value for a concept is simply its $\Sigma \mathrm{Cf}$ value divided by its number of features (NOF). Similarly, the concepts average ff value is its $\Sigma \mathrm{ff}$ value divided by the maximum number of possible connections among the features; that is, $\left(\mathrm{NOF}^{2}+\mathrm{NOF}\right) / 2$.

The strong positive correlations between $\Sigma \mathrm{ff}$ and NOF (and NOA) provide evidence that features are indeed interconnected. This interconnectivity indicates that features are not independent of each other; instead, they refer to each other, as well as to the concept. In addition, the strong direct relationship between $\mathrm{ff}$ and NOA leads to the conclusion that semantic relatedness increases with complexity.

Additional measures and correlations resulting from Experiment 1 will be reported below when relevant to the later experiments.

\section{EXPERIMENT 2}

In order to make Experiment 2 more readable, it will be separated into Experiments $2 \mathrm{~A}$ and $2 \mathrm{~B}$. The semantic congruency task of Experiment $2 \mathrm{~A}$ is of central interest. Here we test the basic prediction of the Klimesch model. That is, contrary to the usual fan effect, concepts with many features are expected to be judged more quickly than those with only a few features. The lexical verification task of Experiment 2B allows for the measurement of incidental factors that affect decision times, such as familiarity (e.g., McCloskey, 1980). The Method section will first describe Experiment $2 \mathrm{~A}$ and will then describe how Experiment 2B differs.

\section{Method}

\section{Experiment 2A: Semantic Congruency Task}

Subjects. The subjects were 36 students in introductory psychology and rhetoric classes at the University of California, Davis, who received extra credit for participating in the experiment. One subject who made a large number of classification errors was replaced.

Material. For the experimental trials, 16 concept words were selected for each of the same six superordinate categories used in Experiment 1. Each of the resulting 96 concept words was paired with a feature word in such a way that for a given superordinate category, eight such concept-feature word pairs were congruent and eight were incongruent. A congruent pair is one in which the feature word represents a feature, property, or attribute of the paired concept; for example, within the superordinate category BIRDS, the concept-feature pair LARK-SINGS would be congruent, and the pair CANARY-BLUE would be incongruent. The 48 concepts used in the congruent pairs were the same concepts measured in Experiment 1 .

In addition to the NOF and NOA measures of the number of core features discussed above, this experiment also controlled for the strength of the connection between the concept and the particular feature used in congruent pairs. The frequency-of-association (FOA) value of a given feature for a given concept represents the percentage of subjects who listed that particular feature when asked to list characteristic features of that concept. These values were obtained at the same time as the NOF values (Klimesch, 1987).

The congruent pairs were selected in such a way as to separate effects of NOA, or NOF, from effects of FOA. That is, half of the concepts with high NOA and NOF values were paired with high FOA properties, while the other half were paired with low FOA properties; and similarly with concepts having low NOA and NOF values.

For the congruent word pairs used in this experiment, the low, average, and high values, together with the standard deviations, for each of the three measures are presented in Table 2. As an example of a word pair used in this experiment, consider the conceptfeature pair PEAR-sweET. Toglia and Battig (1978) reported that the average NOA rating for PEAR was 3.02, and Klimesch (1987) found that the number of different features listed for the concept Pear was 3. Thus Pear is on the low end of both the NOA and the NOF values for the concepts used in this experiment. The feature swEet had been given in response to PEAR by $39.3 \%$ of the subjects whom Klimesch tested. This is near the middle of the FOA values for the feature-concept pairs used in this experiment. For the concept and feature words used in this experiment, the FOA values correlated -.221 with the NOA values and -.042 with the NOF values.

Table 2 also presents the descriptive statistics for several additional measures of the congruent concepts. The $\Sigma$ ff values, discussed 
Table 2

Characteristics of the Word Pairs Used in the Experiments

\begin{tabular}{lcccr}
\hline & Lowest & Average & Highest & \multicolumn{1}{c}{$S D$} \\
\hline NOA & 2.77 & 3.82 & 5.74 & .58 \\
FOA & 14.3 & 44.1 & 96.4 & 19.00 \\
Cf & 2.64 & 4.49 & 5.67 & .70 \\
Iff & 1.03 & 35.87 & 106.21 & 26.89 \\
CLx & 532.44 & 671.08 & 940.22 & 76.81 \\
flx & 567.87 & 660.40 & 831.31 & 62.96 \\
\hline
\end{tabular}

Note-NOA, number of attributes (Toglia \& Battig, 1978); FOA, frequency of association (Klimesch, 1987); $\mathrm{Cf}_{\mathrm{T}}$, rating obtained in Experiment 1 , of connection strength between concepts and features used in Experiments 2-4; $\Sigma \mathrm{ff}$, total of average ratings of connection strength between pairs of features; CLx, lexical verification time to concept words in Experiment 2B; fLx, lexical verification time to feature words in Experiment 2B.

with Experiment 1, may be a much better measure of the strength of concept interconnectivity than were the NOA and NOF measures originally used to design this experiment. PEAR, incidentally, has a $\Sigma$ ff value of 7.17 , well below the mean of 35.87 . Another measure, also obtained in Experiment 1, is the rating of the direct connection between the concept and the congruent feature used in Experiment 2, $\mathrm{Cf}_{\mathrm{T}}$. Although this $\mathrm{Cf}_{\mathrm{T}}$ rating might be thought to measure the same type of relationship as does FOA, it measures it in a very different way, and the correlation over the word pairs used in this experiment is not all that high $(r=.222)$; but it correlated even less with NOF $(r=.105)$ and $\Sigma$ ff $(r=.161)$. The example PEAR, which scores near the middle of the FOA range, also received a score (4.60) near the middle of the $\mathrm{Cf}_{\mathrm{T}}$ range (4.487). The other measures reported in Table 2 will be discussed with Experiment 2B.

Before the actual experiment, the subjects were given practice trials with a different set of six superordinate categories having a total of 24 concept-feature pairs, half congruent and half incongruent.

Word pairs used in both practice and experimental trials had been pretested on an earlier group of 12 subjects to ensure that the words would be correctly understood by almost all subjects under these conditions. The list of stimulus materials is presented in Appendix C.

Design. The subjects were assigned to either the concept-feature group or the feature-concept group according to a predetermined schedule, in such a way that there were 18 subjects in each group. The subjects in the concept-feature group began each trial by seeing a concept word before the paired feature word. Those in the feature-concept group saw the feature word before the concept word.

To increase the likelihood that subjects would properly interpret the meaning of each word (e.g., SwALLow as a BIRD rather than as an activity), words were blocked. This blocking was done by pairs of superordinate categories, rather than blocking by a single superordinate, in order to remove the possibility that subjects in the feature-concept group might occasionally respond on the basis of a feature before seeing the concept. For example, if BIRDS had not been blocked with FRUITS, a feature-concept subject could have responded "congruent" immediately upon seeing wINGs, without needing to wait for the concept word RoBIN. All concept words and feature words within a block fit within one of the two superordinate categories given for that block, but in incongruent pairs, the feature did not fit that particular concept. No concept word or feature word was used more than once for any given subject. At the beginning of a block, the subjects were told which two superordinate categories had been used to generate the concepts in that block. The order of these blocks was counterbalanced across subjects, and the order of concept-feature pairs within blocks was different for each subject.
The practice blocks were TOOLS and UTENSILS, ANIMALS and FURNITURE, and CONTAINERS and WEATHER.

Procedure. The subjects were tested individually in front of a display screen of a personal computer that controlled the presentation of the words and measured each subject's classification response and VT to the nearest $2 \mathrm{msec}$. The procedure will be described for the subjects in the feature-concept group. The procedure for the subjects in the concept-feature group was identical, except for the ordering of concept and feature words.

At the beginning of a block of trials, each subject was told the two superordinate categories that contained all of the concept words in the coming block. Then, at the beginning of a trial, the word "Property:," followed $.5 \mathrm{sec}$ later by a feature word, would appear for $1.5 \mathrm{sec}$ near the center of the screen-for example, "Property: COLLAR." Then the word "Concept:," followed $.5 \mathrm{sec}$ later by a concept word, would appear three rows belowfor example, "Concept: SHIRT." Thus, the time between the presentation of the first critical word (e.g., "COLLAR") and the second critical word (e.g., "SHIRT") was $2 \mathrm{sec}$. These four words then remained on the screen until the subject responded. The screen was then empty for $3 \mathrm{sec}$ before the next feature word.

The subjects responded with a two-way toggle switch, moving it in one direction for congruent pairs and in the other direction for incongruent pairs; they made their decisions on the basis of whether the feature and concept would typically go together, even though a counterexample might be found. They were given the example of the concept of WoLF, and they were told that the properties HOWLS, FUR, TEETH, LEGS, RUN, and STRONG were examples of congruent properties, whereas the properties PURRS, SCALES, GILLS, WINGS, SWIMS, and WEAK were examples of incongruent ones. "Even though a particular wolf might on occasion swim or be weak, we do not typically associate the latter two properties with the concept WoLF." They were instructed to respond as quickly as possible while still making few mistakes. They were told that the decisions would not be tricky, and that the purpose of the experiment was not to see how one makes difficult or tricky decisions, but rather how rapidly one can make easy ones.

The subjects were given feedback during the practice trials, but not during the experimental trials.

\section{Experiment 2B: Lexical Verification Task}

Subjects. The subjects were a separate set of 36 students in introductory psychology and rhetoric classes at the University of California, Davis, who received extra credit for their participation.

Material. The same words were used as in Experiment 2A. However, only the concept and feature words from the previous congruent pairs were left in word form. Words from the incongruent pairs were modified so that, although they continued to look like words (and continued to be pronounceable), they were no longer English words. For example, PISTOL became POSTIL.

Procedure. The task was to determine quickly whether or not a letter string was a common English word. At the beginning of a trial, the word "Stimulus:" appeared for $1 \mathrm{sec}$ near the center of the screen. Then the letter string would appear to the right-for example, "Stimulus: SHIRT." This remained on the screen until the subject responded. The screen would then be empty for $3 \mathrm{sec}$ before the next stimulus. The subjects were again tested individually in front of a monitor of a computer that controlled the stimulus presentation and recorded each subject's response and VT. The subjects responded by means of a two-way toggle switch, moving it in one direction for words and in the other direction for nonwords. They were told that any word would be an extremely common English word; they would not have to search their vocabularies for any unfamiliar words. The subjects were given feedback during the practice trials, but not during the experimental trials.

Design. The subjects were assigned to either the concept group or the feature group according to a predetermined schedule, in such 
a way that there were 18 subjects in each group. The subjects in the concept group saw only letter strings from the concept words. Those in the feature group saw only letter strings from the feature words.

\section{Results}

Except where explicitly noted, the results reported below refer to the semantic congruency task of Experiment $2 \mathrm{~A}$. The alpha level for all analyses was set at .05 .

The analyses were performed both on uncorrected VTs and on normalized ( $z \mathrm{VT}$ ) scores. To obtain the $z \mathrm{VT}$ scores, all of a subject's correct congruent responses were normalized and any score greater than two standard deviations from the subject's mean was reduced to a $z$ score of two, with the appropriate sign. The analyses performed on normalized scores were performed mainly for comparison with the results of Experiments 3 and 4. The 36 subjects included in the analyses made relatively few errors. The VTs of error responses were not included in any of the analyses. The two word pairs associated with the highest error probabilities were MoTOR-SCOOTER, classified as incongruous by $44 \%$ of the feature-concept subjects, and CUCUMBER-SEEDS, classified as incongruous by $44 \%$ of the concept-feature subjects. The pattern of results is not changed if these two pairs are deleted from the analyses. Even when these pairs are included, the average number of errors is less than 2 subjects/pair and less than 2.5 pairs/subject in each of the groups. Congruent word pairs that resulted in a greater number of errors also resulted in longer correct reaction times $(r=.459$ and .523 in the concept-feature and feature-concept conditions, respectively). The subjects in Experiment 2B made an average of $5.9 \%$ errors in each of the groups.

Two types of analyses of variance were performed. In the first, word pairs were treated as the random factor; in the second, subjects were treated as the random factor.

With word pairs used as the random factor, the pairs were divided to produce a $2 \times 2 \times 2$ design, with high versus low NOA values crossed with high versus low FOA values, and with each pair tested under conceptfeature and feature-concept conditions. Eight pairs were dropped because they did not fit cleanly into the classification. These were pairs with NOA and/or FOA scores very near the overall average value, and they are starred in Appendix C. ${ }^{5}$
The average NOA, FOA, and VT for each of these sets of word pairs under both conditions are presented in Table 3 , along with the average $z \mathrm{VT}$ and the error percentage.

The analysis of raw VT scores showed that the featureconcept order resulted in slower VTs than did the concept-feature task $\left[F(1,36)=52.01, M S_{\mathrm{e}}=6,558.28\right]$. Freedman and Loftus (1971) also found such VTs to be faster when a category was given before an adjective than vice versa. Their explanation was that when the adjective is given first, activation can spread to unrelated categories that are not highly interconnected. In fact, one might wonder why a reversed fan effect, based on the NOA measure obtained from the concepts, would be expected from the feature-concept order. There are two possibilities, both of which would also predict the longer VTs with the feature-concept order. First, the explanation based on Klimesch's (1987, 1988, in press) connectivity theory suggests that the most important spread of activation begins not with the presentation of the first word, but rather with the presentation of the concept word (thus resulting in longer VTs). That is, when the feature is presented first, the activation spreads among so many unrelated, loosely connected categories that it has relatively little effect on the ultimate decision compared to that of the relatively compact (highly interconnected) network resulting from the presentation of the concept. Another possible explanation might be built upon the potential context effect resulting from the earlier presentation of the name of the superordinate category and from the blocking of similar concepts (e.g., McCloskey \& Glucksberg, 1979). This context effect, in turn, may prime those concept networks within the superordinate category so that the correct concept network is more likely to be activated than would be the case without such priming. Nevertheless, one would still expect, with the latter explanation, a wider spread among less highly interconnected networks from the feature-concept order than from the conceptfeature order. This wider spread would explain the longer VTs, but it is difficult to see why it would result in virtually the exact same pattern of VTs as is obtained with concept-feature order without involving the first explanation as well.

The VTs were faster with high NOA pairs than with low NOA pairs $\left[F(1,36)=4.526, M S_{e}=29,678.44\right]$;

Table 3

Average NOA, FOA, and Verification Time (in Milliseconds) for Each Set of Congruent Word Pairs for Each Group, With Pairs as the Random Variable

\begin{tabular}{|c|c|c|c|c|c|c|c|c|c|}
\hline \multirow{2}{*}{\multicolumn{4}{|c|}{ Congruent Word Pair Sets }} & \multicolumn{6}{|c|}{ Group } \\
\hline & & & & \multicolumn{3}{|c|}{ Concept-Feature } & \multicolumn{3}{|c|}{ Feature-Concept } \\
\hline NOA & FOA & NOA & FOA & VT & $z \mathrm{VT}$ & $\%$ Error & VT & $z \mathrm{VT}$ & \% Error \\
\hline low & low & 3.42 & 32.3 & 1,002 & .19 & 10.6 & 1,115 & .18 & 13.9 \\
\hline low & high & 3.23 & 60.0 & 907 & -.12 & 10.6 & 1,118 & .16 & 8.9 \\
\hline high & low & 4.41 & 30.4 & 904 & -.15 & 7.8 & 1,012 & -.15 & 7.2 \\
\hline high & high & 4.14 & 60.7 & 904 & -.14 & 7.2 & 993 & -.12 & 5.0 \\
\hline
\end{tabular}

Note-NOA, number of attributes; FOA, frequency of association; VT, congruency verification time; $z \mathrm{VT}$, normalized verification time. 
Table 4

Average NOA, FOA, and Congruency Verification Time (in Milliseconds) for Congruent High and Low NOA Word Pairs for Each Group, With Subjects as the Random Variable

\begin{tabular}{|c|c|c|c|c|c|c|c|c|}
\hline \multirow[b]{3}{*}{ Pairs } & \multirow[b]{3}{*}{ NOA } & \multirow[b]{3}{*}{ FOA } & \multicolumn{6}{|c|}{ Group } \\
\hline & & & \multicolumn{3}{|c|}{ Concept-Feature } & \multicolumn{3}{|c|}{ Feature-Concept } \\
\hline & & & VT & $\mathbf{L x}$ & $\%$ Error & VT & $\mathbf{L x}$ & \%6 Error \\
\hline \multicolumn{9}{|l|}{ Congruent } \\
\hline Low NOA & 3.52 & 43.8 & 986 & 697 & 12.5 & 1,137 & 643 & 12.3 \\
\hline High NOA & 4.12 & 44.9 & 886 & 656 & 6.9 & 989 & 678 & 6.5 \\
\hline Incongruent & & & 1,062 & & 11.7 & 1,144 & & 10.4 \\
\hline
\end{tabular}

Note-NOA, number of attributes; FOA, frequency of association; VT, verification time; $L X$, average lexical verification time.

that is, decisions on concepts with a large number of features are made more rapidly than on those with fewer features. This was also found with the $z \mathrm{VT}$ measure $[F(1,36)$ $\left.=4.395, M S_{e}=0.273\right]$. There was no discernible effect of FOA, and none of the interactions were significant with either measure.

With subjects used as the random factor, each subject's VTs were averaged separately over word pairs with concepts above the average NOA value for the relevant superordinate category, and over pairs with concepts below the average NOA value for the relevant superordinate category. The pairs had been originally selected so that this separation would result in equal FOA averages for the two sets. This equality, however, was based on all the experimental pairs and the discarded erroneous decisions could have caused large differences between the FOA averages for high and low NOA sets for any particular subject. However, the greatest difference observed in FOA averages between the two sets was $4.3 \%$, and the average absolute difference across all subjects was $1.6 \%$.

The resulting VT averages formed a $2 \times 2$ (NOA level $x$ feature/concept order) analysis, with NOA within subjects and order between subjects. The effect of FOA was not tested, but was equated across NOA levels. This means that any FOA effect would increase the error term and, thus, would reduce the power of the analysis. The average NOA, FOA, and VT for each of these sets of pairs under both conditions are presented in Table 4 . Only the VT difference between NOA levels is significant $[F(1,34)$ $\left.=47.17, M S_{\mathrm{c}}=5,857.46\right]$, not the difference between orders $\left[F(1,34)=2.288, M S_{e}=126,801.31\right]$, or the interaction $\left[F(1,34)=1.770, M S_{e}=5,857.46\right]$.

Table 5 presents the correlations of the VTs, averaged across subjects for each congruent pair (over all 48 pairs), with the NOA and $\Sigma$ ff values of the concept words, with the FOA and $\mathrm{Cf}_{\mathrm{T}}$ values of the pairs, with the lengths of predicate and concept words, and with lexical VTs from Experiment 2B for concept words and for feature words. The negative correlations indicate that VTs are faster with larger NOA or FOA values. The NOA values correlate significantly with VTs of each of the groups and with the times averaged over both groups. The FOA values, on the other hand, correlate significantly only with VTs of the concept-feature group.
One difficulty with experiments involving the selection of words with different characteristics, rather than the manipulations of words such that the same words can be used in all conditions, is that one is never certain that the selection procedure has not contained a biasing factor. In

Table 5

Correlations and Partial Correlations of Pair Verification Times With the NOA and Iff Values of the Concept Words, the FOA and $\mathrm{Cf}_{\mathrm{T}}$ Values of the Feature-Concept Pairs, the Length of the Concept and Feature Words, and the Lexical Verification Times for Concept and Feature Words

\begin{tabular}{|c|c|c|c|}
\hline & \multicolumn{2}{|c|}{ Group } & \multirow[b]{2}{*}{ Average } \\
\hline & Concept-Feature & Feature-Concept & \\
\hline \multicolumn{4}{|c|}{ Correlations $(d f=46)$} \\
\hline NOA & $-.347 \dagger$ & $-.433 \ddagger$ & $-.435 \ddagger$ \\
\hline$\Sigma$ ff & $-.337 \dagger$ & $-.428 \dagger$ & $-.428 \dagger$ \\
\hline FOA & $-.305^{*}$ & -.123 & -.215 \\
\hline $\mathrm{Cf}_{\mathrm{T}}$ & $-.349 \dagger$ & $-.366 \dagger$ & $-.392 \dagger$ \\
\hline CLn & .172 & $.450 \ddagger$ & $.370 \dagger$ \\
\hline fLn & .033 & .006 & .019 \\
\hline CLx & $.337 \dagger$ & $.483 \ddagger$ & $.463 \ddagger$ \\
\hline fLx & .008 & -.089 & -.055 \\
\hline \multicolumn{4}{|c|}{ Partial Correlations $(d f=45)$} \\
\hline NOA.FOA & $-.446 \ddagger$ & $-.475 \ddagger$ & $-.506 \ddagger$ \\
\hline FOA.NOA & $-.419 \ddagger$ & -.249 & $-.354 \dagger$ \\
\hline NOA.CLn & $-.308^{*}$ & $-.318^{*}$ & $-.343 \dagger$ \\
\hline FOA.CLn & $-.315^{*}$ & -.148 & -.239 \\
\hline NOA.fLn & $-.355 \dagger$ & $-.438 \ddagger$ & $-.441 \ddagger$ \\
\hline FOA.fLn & $-.306 *$ & -.124 & -.215 \\
\hline NOA.CLX & $-.316^{*}$ & $-.413 \ddagger$ & $-.414 \ddagger$ \\
\hline FOA.CLX & -.255 & -.023 & -.246 \\
\hline NOA.fLx & $-.372 \ddagger$ & $-.430 \ddagger$ & $-.444 \ddagger$ \\
\hline FOA.fLX & $-.322 *$ & -.023 & -.246 \\
\hline$\Sigma \mathrm{ff} . \mathrm{Cf}_{\mathbf{T}}$ & $-.304^{*}$ & $-.402 \ddagger$ & $-.401 \ddagger$ \\
\hline $\mathrm{Cf}_{\mathrm{T}} \cdot \mathbf{\mathrm { Iff }}$ & $-.317^{*}$ & $-.333 \dagger$ & $-.362 \dagger$ \\
\hline \multicolumn{4}{|c|}{$\begin{array}{l}\text { Double Partial Correlations With Both Length } \\
\text { and Lexical }(d f=44)\end{array}$} \\
\hline NOA.concept & $-.313^{*}$ & $-.331^{*}$ & $-.357 \dagger$ \\
\hline FOA.concept & -.256 & -.029 & -.141 \\
\hline NOA.feature & $-.379 \ddagger$ & $-.435 \ddagger$ & $-.450 \ddagger$ \\
\hline FOA feature & -.253 & -.021 & -.131 \\
\hline
\end{tabular}

Note-NOA, number of attributes; $\Sigma \mathrm{ff}$, total of average ratings of connection strength between pairs of features; FOA, frequency of association; $\mathrm{Cf}_{\mathrm{T}}$, rating of connection strength between concepts and features used in Experiments 2-4;CLn, concept length; fLn, feature length; $C L x$, lexical verification time to concept words; $\mathrm{fLx}$, lexical verification time to feature words. ${ }^{*} p<.05 . \quad \dagger p<.02 . \quad \ddagger p<.01$ 
Table 6

Multiple Linear Regressions Based on the Concept -Feature Verification Times, the Feature-Concept Verification Times, and the Average of the Two

\begin{tabular}{lccr}
\hline \multirow{2}{*}{$\begin{array}{l}\text { Predictor } \\
\text { Variable }\end{array}$} & \multicolumn{3}{c}{ Beta Weights } \\
\cline { 2 - 4 } & Concept-Feature & Feature-Concept & Average \\
NOA & Predicting With NOA and FOA & \\
FOA & -.414 & -.323 & -.392 \\
CLx & -.347 & -.132 & -.238 \\
CLn & .233 & .343 & .326 \\
fLx & -.061 & .211 & .111 \\
fLn & .031 & -.005 & .011 \\
$R$ & -.054 & -.029 & -.042 \\
& & & \\
& $.562 *$ & $.645 \dagger$ & $.642 \dagger$ \\
$\Sigma f f$ & & & \\
Cf & Predicting With $\Sigma$ ff and Cf & \\
CLx & -.207 & -.195 & -.218 \\
CLn & -.339 & -.310 & -.351 \\
fLx & .297 & .360 & .365 \\
fLn & -.035 & .223 & .130 \\
$R$ & .075 & -.004 & .030 \\
$R$ & -.022 & -.012 & -.018 \\
\hline
\end{tabular}

Note-NOA, number of attributes; FOA, frequency of association; CLx, lexical verification time to concept words; $C L n$, concept length; $f L x$, lexical verification time to feature words; fLn, feature length; $\Sigma$ ff, total of average ratings of connection strength between pairs of features; $\mathrm{Cf}_{\mathrm{T}}$, rating of connection strength between concepts and features used in Experiments 2-4. $\quad d f=6,41 . \quad{ }^{*} p<.05 . \quad \dagger p<.005$.

this experiment, it would seem to be most important to equate FOA values-well known for their importanceacross conditions and to partial out this variable from the NOA effect, which is most important for the testing of the present hypothesis. Note that the partial correlations of NOA.FOA with VTs for each of the groups and averaged over groups are highly significant.

For further exploration of the possibility that the NOA effect might be caused by a sampling bias, Table 5 also includes correlations between NOA and the VTs with the following effects partialed out: (1) length of the concept word, (2) length of the feature word, (3) lexical VTs for concept words, (4) lexical VTs for feature words, (5) both the length and lexical VTs to concept words, and (6) both the length and lexical VTs for feature words. (The lexical VTs were obtained from Experiment 2B.) These partial correlations are also reported for FOA effects for purposes of comparison. As is shown in Table 5, the correlations between NOA and pair VTs remain significant, regardless of which other effect is partialed out. Although the possibility always remains that some unconsidered, and hence uncontrolled, variable may be the "real cause" of the observed NOA effect, the variables with the most obvious potential for doing so (FOA, word length, and such factors as frequency and familiarity, which should be major influences on lexical VTs) do not seem to detract from the NOA effect.

The measures obtained in Experiment $1, \Sigma \mathrm{ff}$ and $\mathrm{Cf}_{\mathrm{T}}$, also have significant negative correlations with the conceptfeature pair verification times.
Table 6 presents the beta weights obtained with the multiple linear regressions when the dependent variable was the standardized pair VTs for each of the groups and for the average over both of the groups. The first column represents beta weights when VTs were used from the concept-feature group, the second column when VTs were used from the feature-concept group, and the third column when VTs were averaged over both groups. At the top of the table, NOA and FOA are used in the prediction equation; at the bottom of the table, $\Sigma \mathrm{ff}_{\text {and }} \mathrm{Cf}_{\mathrm{T}}$ are used in the prediction equation. Thus, the multiple correlation for the feature-concept group, when predicting with NOA and FOA, was .645 and was obtained with standardized NOA values weighted at -.323 , the standardized FOA values at -.132, the standardized lexical VTs for the concept word (from Experiment 2B) weighted at .343, etc.

The correlations, partial correlations, and weights for the multiple correlations fit the hypothesis that the number of features closely tied to a concept is a good predictor of how rapidly a person can decide whether or not any particular feature belongs to that concept. These correlations also fit the hypothesis that a concept's number of features is at least as good a predictor of a person's VT as is the degree of association between the concept and the judged feature, as measured by the FOA values for that concept. The NOA correlations with VT are significantly less than zero.

\section{EXPERIMENT 3}

In order to demonstrate that the reversed fan pattern of VTs is a function of the processing involved in a semantic task - either a category membership task (Klimesch, 1987 ) or a category-feature congruency task (Experiment 2A)-in Experiment 3, we used the same word set in a retrieval task following laboratory learning ("memory for episodic encodings"). Previous experiments had demonstrated that a great variety of tasks involving retrieval of episodic learning result in the fan effect. The effect of the semantic complexity on the retrieval of episodic information is more complicated and related to the degree to which the retrieval task encourages the subject to use the semantic relationships while searching episodic memory (e.g., Nelson, Canas, \& Bajo, 1987; cf. Dosher \& Rosedale, 1991).

If NOA indeed measures the number of attributes branching off from a concept node, a retrieval task should result in either the typical fan effect (if the subject attempts to use the semantic relationships to retrieve the episodic information) or no fan effect (if the subject does not attempt to use the semantic relationships). If, however, the result pattern of Experiment $2 \mathrm{~A}$ is due to some peculiarity of the word set, a reversed fan effect would be expected here as well.

\section{Method}

Subjects. The subjects were 48 students in introductory psychology and rhetoric classes at the University of California, Davis, who received extra credit for participating. 
Design. During the study stage, the subjects were told to observe and remember the concept-feature pairs. They were further told that their memory would be better if they looked for a meaningful relationship between the paired words. The subjects saw the stimuli in the same way as did the concept-feature subjects in Experiment $2 \mathrm{~A}$. That is, a block of trials began with the presentation of the names of the categories from which the concepts within that block were chosen. At the beginning of each study trial, the word "Concept:" appeared on the screen, followed in .5 sec by one of the concept words, which was followed $1.5 \mathrm{sec}$ later by the word "Property:" and .5 sec later by the congruent feature. These words remained on the screen for $5 \mathrm{sec}$ before the screen went blank for $1 \mathrm{sec}$, followed by the next concept.

Half the concepts were presented only once, with a single feature word: half in the first half of the block (1F-study), and the remainder in the second half (1S-Study). In order to create an episodic fan, the other half of the concepts were seen twice, with a different feature word each time. The subjects had been told that this would occur and that, in these cases, they should remember both feature words paired with the concepts. For half of these concepts, the feature word actually tested was the one presented in the first half of the block (2F-Study), and for the other half, it was the one in the second half of the block (2S-Study). Thus, the number indicates the number of features seen with a particular concept (one or two), and the letter indicates whether the tested conceptfeature pair had occurred during the first or second half of study.

In all cases, the pairs that would be targets in the test stage were the same as the congruent pairs of Experiment 2A. An additional eight pairs per block became foils in the test stage. The concepts for the foils were chosen from the incongruent pairs of Experiment $2 \mathrm{~A}$, but were re-paired with congruent properties. The presentation order of the word pairs and the assignment of words to conditions (1F-, 1S-, 2F-, and 2S-Study) were different for each subject in such a way that all target pairs were used equally often in all four conditions.

Immediately after each study stage, the subject received the test stage for this block. In the test stage, the subject received all 16 of the target pairs, plus the 8 foils, in which the concept-feature pairing was not the same as it had been during the study stage. (That is, the foils, like the targets, were congruent during both study and test phases, but, unlike the targets, the foil concepts were paired with different features during the test phase than they had been during the study phase.) A test trial again presented the concept word, followed by the feature word (with the same 2.5-sec time between stimuli that was used in Experiment 2A), and the subject decided as quickly as possible if this pair had been presented during the study trial. The subjects were told to respond as quickly as possi- ble while keeping the error rate low. They were also told that it was more important to reject false positives than to score hits. The subjects received feedback during the practice blocks, but not during the experimental blocks.

\section{Results}

For Experiment 3 analyses, each subject's recognition times were normalized over all of the subject's correct responses, and any score greater than two standard deviations from the subject's mean was reduced to a $z$ score of two, with the appropriate sign. This was done to reduce the influence of the differences in overall recognition times between subjects, which otherwise would have been much greater in this experiment than it was in Experiment 2 because of two factors. First, not all word pairs were seen by all subjects in all study conditions, and thus, because the the random assignment, some words were seen in some conditions primarily by slow subjects, and in other conditions primarily by fast subjects. Second, this first factor was exacerbated by a larger error rate $(18.6 \%)$ in this experiment.

Table 7 presents the average normalized recognition times $(z R T)$ for the same $\mathbf{4 0}$ word pairs that were analyzed in Experiment 2A. The data were averaged across subjects, and word pairs were treated as the random variable in a $2 \times 2 \times 2 \times 2$ analysis of variance (NOA level $\times$ FOA level $x$ one-two presentations $\times$ first-second half). The analysis showed that high FOA pairs were recognized faster than low FOA pairs $\left[F(1,36)=11.881, M S_{e}=\right.$ .237], concepts learned with one feature were recognized faster than those learned with two $\left[F(1,36)=35.130, M S_{e}\right.$ $=.131]$, and pairs seen in the first half of the block were recognized faster than those seen in the second half $[F(1,36)$ $=4.559$ ]. However, the latter effect was qualified by a one-two $\times$ first-second interaction $[F(1,36)=11.617]$, with the first-second half effect being present only for the concepts presented twice, with two different properties. There was no overall effect of NOA $[F(1,36)<1]$, nor did the NOA effect interact significantly with the other variables.

Table 7

Average Recognition Time (Normalized) for Each Set of Target Word Pairs for Each Study Condition in Experiment 3, With Pairs as the Random Variable

\begin{tabular}{|c|c|c|c|c|c|c|c|c|}
\hline \multirow{2}{*}{\multicolumn{2}{|c|}{ Word Pair Set }} & \multicolumn{7}{|c|}{ Recognition Time } \\
\hline & & \multicolumn{4}{|c|}{ Study Condition } & \multicolumn{3}{|c|}{ Averaged over F-S } \\
\hline NOA & FOA & $1 \mathrm{~F}$ & is & $2 F$ & $2 S$ & Once & Twice & All \\
\hline low & low & -.177 & -.237 & .140 & .454 & -.207 & .282 & .037 \\
\hline low & high & -.304 & -.250 & -.023 & .263 & -.277 & .120 & -.078 \\
\hline high & low & .112 & .117 & .235 & .442 & .115 & .338 & .227 \\
\hline \multirow[t]{3}{*}{ high } & high & -.247 & -.376 & -.224 & .093 & -.311 & -.065 & -.188 \\
\hline & low & & & & & & & .132 \\
\hline & high & & & & & & & -.133 \\
\hline low & & & & & & -.242 & .201 & -.020 \\
\hline high & & & & & & -.098 & .137 & .020 \\
\hline All & & -.154 & -.187 & .032 & .305 & -.170 & .032 & \\
\hline
\end{tabular}

Note-NOA, number of attributes; FOA, frequency of association; IF, the concepts presented only once, with a single feature word, in the first half of the block; $1 S$, the concepts presented only once, with a single feature word, in the second half of the block; $2 \mathrm{~F}$, two feature words presented in the first half of the block; $2 \mathrm{~S}$, feature words presented in the second half of the block. 
Table 8

Correlations of Nermalized Recognition Times With NOA Values of the Concept Words and FOA Values of the Concept-Feature Pairs

\begin{tabular}{lcccccccc}
\hline & \multicolumn{4}{c}{ Study Condition } & & \multicolumn{3}{c}{ Averaged Over F-S } \\
\cline { 2 - 4 } \cline { 7 - 9 } & $1 \mathrm{~F}$ & $1 \mathrm{~S}$ & $2 \mathrm{~F}$ & $2 \mathrm{~S}$ & & Once & Twice & All \\
\hline NOA & .066 & .092 & .048 & -.013 & .148 & .031 & .086 \\
FOA & $-.318^{*}$ & -.234 & -.284 & $-.387 \dagger$ & $-.521 \dagger$ & $-.483 \dagger$ & $-.648 \dagger$ \\
\hline
\end{tabular}

Note-NOA, number of attributes; FOA, frequency of association; IF, the concepts presented only once, with a single feature word, in the first half of the block; 1S, the concepts presented only once, with a single feature word, in the second half of the block; $2 \mathrm{~F}$, two feature words presented in the first half of the block; $2 \mathrm{~S}$, feature words presented in the second half of the block. ${ }^{*} p<.05$. $t p<.01$.

Thus, although NOA was shown to be a potent independent variable with the semantic congruency task of Experiment $2 \mathrm{~A}$, it appeared to be ineffective in affecting a subject's $z R T$. The analysis of variance showed that the two most potent independent variables in this task were FOA level and number of properties, paired with a concept within the experiment.

A similar analysis with percent error as the dependent measure showed only the main effect of FOA to be significant $\left[F(1,36)=7.701, M S_{\mathrm{e}}=420.255\right]$, with more errors made with the low FOA pairs $(23.1 \%)$ than with the high FOA pairs (14.1\%).

Table 8 presents the correlations, based on all 48 target pairs, between the $z$ RTs and the NOA and FOA scores. The $z$ RTs display a strong negative correlation with the pair FOA value-as did VTs of Experiment 2A (see Table 5). Now, however, there is no negative correlation with NOA.

The results from Experiment 3, then, give no indication of a reversed fan effect associated with NOA values with the retrieval task, as would be expected if the reversed fan effect in the semantic task of Experiment $2 \mathrm{~A}$ had been caused by the selection of words. In addition, there is also no strong, consistent evidence that NOA causes the typical fan effect in this retrieval task, suggesting that subjects were not using the semantic relationships to search episodic memory. However, this lack of a fan effect associated with NOA may be partially due to a masking from the much stronger fan effect caused by the number of features episodically paired with a concept. Experiment 4 was run partially to remove this potentially masking effect and partially to replicate the episodic fan and semantic reversed fan effects on a single set of subjects.

In Experiment 4, subjects first performed the semantic congruency task, providing a replication of Experiment $2 A$. Then, without prior warning, they were asked to perform an episodic recognition task on the same words, measuring the effect of NOA on an episodic task based on the same word pairs that had just been judged in the semantic task.

\section{EXPERIMENT 4}

\author{
Method \\ Subjects. The subjects were 36 students in introductory psychol- \\ ogy and rhetoric classes at the University of California, Davis, who \\ received extra credit for participating.
}

Design. Each subject began with the semantic task used in Experiment $2 \mathrm{~A}$; there were 18 subjects in each of the concept/feature orders. The episodic task was introduced respectively, after each subject had completed the entire semantic task. The subjects were now told that they were to indicate, as quickly as possible, which pairs had been judged in the semantic task.

During the experimental trials, the target pairs consisted of the congruent pairs from the semantic task; the foil pairs consisted of the concepts from the incongruent pairs, but re-paired with the properties in the episodic task so that they were now also congruent. In order to make it less likely that the subjects would discover this relationship between congruent-incongruent semantic task pairs and target-foil semantic task pairs, practice trials of the semantic task contained foils from the previously congruent pairs as well as from the previously incongruent pairs.

The subjects received feedback on the practice trials of both tasks, but not during the experimental trials.

\section{Results}

In order to make the results of Experiment 4 compatible with those of Experiment 3, the normalized $z \mathrm{VT}$ and $z \mathrm{RT}$ scores were of primary focus. The error rate in the semantic task was similar to that found in Experiment $2 \mathrm{~A}$ (7.5\% in the concept-feature group and $8.2 \%$ in the feature-concept group); the error rate in the episodic task was more similar to that found in Experiment 3 (13.7\% in the concept-feature group and $16.1 \%$ in the featureconcept group).

Table 9 presents the average $z$ RTs for the 40 word pairs analyzed in both Experiments $2 \mathrm{~A}$ and 3 (Tables 3 and 7). Note that the normalized scores do not have to sum to 0 because not all 48 pairs were included. In the analysis of variance performed on these data, the word pairs were again treated as the random variable. This analysis yielded a significant main effect of FOA $\left[F(1,36)=14.039, M S_{e}\right.$ $=.929]$, with subjects responding faster to high FOA pairs. There was also a significant main effect of NOA $\left[F(1,36)=7.116, M S_{\mathrm{e}}=.929\right]$, with subjects responding faster when a pair included a high NOA concept; but this was primarily due to the large NOA effect within the semantic task. In the episodic task, this NOA effect was reversed, resulting in a significant NOA $\times$ task interaction $\left[F(1,36)=5.892, M S_{e}=1.652\right]$. The NOA effect also interacted with the concept/feature presentation order $\left[F(1,36)=7.436, M S_{\mathrm{e}}=.439\right]$, in such way that the difference in average response time between low and high NOA words was greater in the feature-concept condition-as it was in Experiment $2 \mathrm{~A}$. 
Table 9

Average Semantic Verification and Episodic Recognition Times (Normalized) for Each Set of Congruent Word Pairs for Both Groups and Both Tasks, With Pairs as the Random Variable

\begin{tabular}{|c|c|c|c|c|c|c|c|c|c|c|}
\hline \multirow{3}{*}{\multicolumn{2}{|c|}{$\begin{array}{c}\text { Word Pair } \\
\text { Set }\end{array}$}} & \multicolumn{6}{|c|}{ Group } & & & \\
\hline & & \multicolumn{3}{|c|}{ Concept-Feature } & \multicolumn{3}{|c|}{ Feature-Concept } & \multicolumn{3}{|c|}{ Average } \\
\hline & & \multicolumn{2}{|c|}{ Semantic } & \multirow{2}{*}{$\begin{array}{c}\text { Episodic } \\
\text { zRT }\end{array}$} & \multicolumn{2}{|c|}{ Semantic } & \multirow{2}{*}{$\begin{array}{c}\text { Episodic } \\
z \mathrm{RT}\end{array}$} & \multicolumn{2}{|c|}{ Semantic } & \multirow{2}{*}{$\begin{array}{c}\text { Episodic } \\
\text { ZRT }\end{array}$} \\
\hline NOA & FOA & zVT & VT & & $z \mathrm{VT}$ & VT & & $z \mathrm{VT}$ & VT & \\
\hline $\begin{array}{l}\text { low } \\
\text { low } \\
\text { high } \\
\text { high }\end{array}$ & $\begin{array}{l}\text { low } \\
\text { high } \\
\text { low } \\
\text { high }\end{array}$ & $\begin{array}{r}.504 \\
.070 \\
-.253 \\
-.665\end{array}$ & $\begin{array}{r}1,011 \\
966 \\
944 \\
893\end{array}$ & $\begin{array}{r}.016 \\
-.711 \\
.787 \\
-.474\end{array}$ & $\begin{array}{r}.863 \\
.121 \\
-.507 \\
-.616\end{array}$ & $\begin{array}{r}1,103 \\
978 \\
908 \\
889\end{array}$ & $\begin{array}{r}.049 \\
.215 \\
.327 \\
-.724\end{array}$ & $\begin{array}{r}.684 \\
.096 \\
-.380 \\
-.640\end{array}$ & $\begin{array}{r}1,057 \\
972 \\
926 \\
891\end{array}$ & $\begin{array}{r}.032 \\
-.232 \\
.556 \\
-.599\end{array}$ \\
\hline \multicolumn{11}{|c|}{ Averaged over FOA } \\
\hline $\begin{array}{l}\text { low } \\
\text { high }\end{array}$ & & $\begin{array}{r}.287 \\
-.459\end{array}$ & $\begin{array}{l}988 \\
920\end{array}$ & $\begin{array}{r}-.347 \\
.157\end{array}$ & $\begin{array}{r}.492 \\
-.561\end{array}$ & $\begin{array}{r}1,041 \\
899\end{array}$ & $\begin{array}{r}.132 \\
-.198\end{array}$ & $\begin{array}{r}.390 \\
-.510\end{array}$ & $\begin{array}{r}1,015 \\
909\end{array}$ & $\begin{array}{l}-.108 \\
-.021\end{array}$ \\
\hline
\end{tabular}

Note-NOA, number of attributes; FOA, frequency of association; VT, verification time; zVT, normalized verification time; $z \mathrm{RT}$, normalized recognition time.

A similar analysis was performed with percent error as the dependent measure. This analysis showed that there were significantly more errors made in the episodic task than in the semantic task $\left[F(1,36)=7.141, M S_{\mathrm{e}}=\right.$ 281.488].

An analysis of the semantic task raw VT scores was consistent with the analysis of $z \mathrm{VT}$ scores. Subjects with the concept-feature order responded faster than did those with the feature-concept order $\left[F(1,36)=14.331, M S_{c}\right.$ $=4,526.33]$; subjects had faster VTs with high NOA pairs than with low NOA pairs $\left[F(1,36)=9.281, M S_{\mathrm{e}}\right.$ $=25,158.31]$; and these two factors interacted with a greater NOA effect in the feature-concept condition. The effect of FOA was not significant $[F(1,36)=2.857]$.

Table 10 presents the correlations, based on all 48 target pairs, of $z \mathrm{VTs}$ and $z \mathrm{RT}$ with the NOA, $\Sigma \mathrm{ff}, \mathrm{FOA}$, and $\mathrm{Cf}_{\mathrm{T}}$ scores of the pairs, and with the $z \mathrm{RTs}$ from Experiment 3 . The last row of the table gives the correlations between semantic task $z$ VTs and episodic task $z$ RTs of Experiment 4. There are significant negative correlations between the FOA and both $z$ VTs and $z$ RTs (replicating the findings of Experiments $2 \mathrm{~A}$ and 3 ), but the NOA scores have significant negative correlations only with the $z \mathrm{VTs}$ (replicating Experiment 2A) and not with the $z$ RTs (replicating Experiment 3). In fact, there is a suggestion that NOA scores may be positively correlated with $z$ RTs.

Table 11 presents beta weights from the multiple regressions with dependent variables of $z$ VTs and $z$ RTs from Experiment 4. The main point of interest is that the beta weights for NOA are negative for the semantic tasks, but positive for the episodic tasks, whereas the weights for FOA are negative for both types of tasks. The story is more complicated if one uses $\Sigma \mathrm{ff}$ and $\mathrm{Cf}_{\mathrm{T}}$, rather than NOA and FOA, in the prediction equations (Part 2). $\Sigma \mathrm{ff}$ behaves similarly to NOA: negative betas for semantic tasks and positive betas for episodic tasks. $\mathrm{Cf}_{\mathrm{T}}$, however, behaves differently than does FOA: negative betas for semantic tasks, but betas very near zero for episodic tasks. In fact, $\mathrm{Cf}_{\mathrm{T}}$ and FOA do not correlate all that highly with one another (.222). If both $\mathrm{Cf}_{\mathrm{T}}$ and FOA are included in the prediction equations (Part 3), not only do the multiple correlation coefficients improve, but when the SPSS stepwise procedure is used to reduce the equations to the essential components, the $\mathrm{Cf}_{\mathrm{T}}$ component drops out of the equation predicting performance in the episodic memory task, but remains one of the essential components of the

Table 10

Correlations of Normalized Semantic Verification and Episodic Recognition Times of Experiment 4 With NOA, NOF, and $\Sigma$ ff Values of the Concept Words, the FOA and $\mathrm{Cf}_{\mathrm{T}}$ Values of the Concept-Feature Pairs, the Episodic Recognition Times of Experiment 3, and Correlations Between Semantic Verification and Episodic Recognition Times

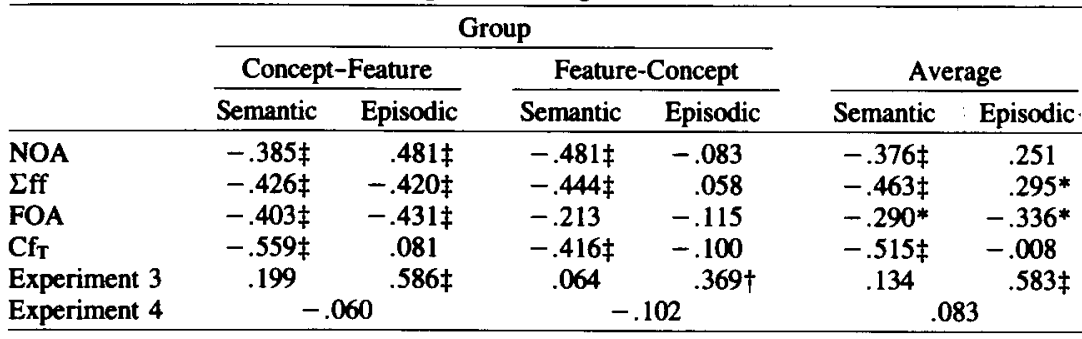

Note-NOA, number of attributes; $\Sigma \mathrm{ff}$, total of average ratings of connection strength between pairs of features; FOA, frequency of association; $\mathrm{Cf}_{\mathrm{T}}$, rating, obtained in Experiment 1 of connection strength between concepts and features used in Experiments $2-4 . *^{*} p<.05$. $\dagger_{p}<$ .02. $\quad \ddagger p<.01$ 
Table 11

Beta Weights From the Multiple Linear Regressions Based on Normalized Concept-Feature, Feature-Concept, and Average Semantic Verification and Episodic Recognition Times of Experiment 4, and on the Average Semantic Verification Times Averaged Over Experiments $2 A$ and 4

\begin{tabular}{|c|c|c|c|c|c|c|c|}
\hline \multirow{3}{*}{$\begin{array}{l}\text { Predictor } \\
\text { Variable } \\
\end{array}$} & \multicolumn{4}{|c|}{ Group } & & & \multirow{3}{*}{$\begin{array}{c}\text { Experiments } 2 B \text { and } 4 \\
\text { Semantic Task } \\
\end{array}$} \\
\hline & \multicolumn{2}{|c|}{ Concept-Feature } & \multicolumn{2}{|c|}{ Feature-Concept } & \multicolumn{2}{|c|}{ Experiment 4 Average } & \\
\hline & Semantic Task & Episodic Task & Semantic Task & Episodic Task & Semantic Task & Episodic Task & \\
\hline \multicolumn{8}{|c|}{ Part 1: Predicting With NOA and FOA } \\
\hline NOA & -.488 & .443 & -.344 & .011 & -.439 & .283 & -.426 \\
\hline FOA & -.504 & -.457 & -.341 & -.200 & -.446 & -.403 & -.315 \\
\hline CLx & .143 & -.235 & .209 & .059 & .187 & -.112 & .300 \\
\hline CLn & -.080 & .057 & .229 & .056 & .086 & .069 & .108 \\
\hline fLx & .020 & -.236 & -.241 & -.325 & -.123 & -.339 & -.030 \\
\hline fLn & -.172 & .023 & -.075 & .041 & -.128 & .039 & -.072 \\
\hline $\boldsymbol{R}$ & $.654^{*}$ & $.657^{*}$ & $.703 *$ & .347 & $.685^{*}$ & .499 & $.677^{*}$ \\
\hline \multicolumn{8}{|c|}{ Part 2: Predicting With $\Sigma$ ff and $\mathrm{Cf}_{\mathrm{T}}$} \\
\hline$\Sigma$ ff & -.312 & .392 & -.203 & .201 & -.272 & .363 & -.245 \\
\hline $\mathrm{Cf}_{\mathrm{T}}$ & -.530 & -.001 & -.332 & -.106 & .454 & -.058 & -.400 \\
\hline CLx & .224 & -.068 & .267 & .164 & .262 & .054 & .351 \\
\hline CLn & -.083 & -.032 & .236 & .060 & .087 & .015 & .123 \\
\hline fLx & .124 & -.025 & -.178 & -.286 & -.035 & -.184 & .011 \\
\hline fLn & -.112 & .087 & -.038 & .061 & -.078 & .090 & -.037 \\
\hline$R$ & $.694^{*}$ & .434 & $.700^{*}$ & .423 & $.700^{*}$ & .364 & $.705^{*}$ \\
\hline
\end{tabular}

Part 3: Predicting with $\Sigma f f, \mathrm{Cf}_{\mathrm{T}}, \mathrm{FOA}$

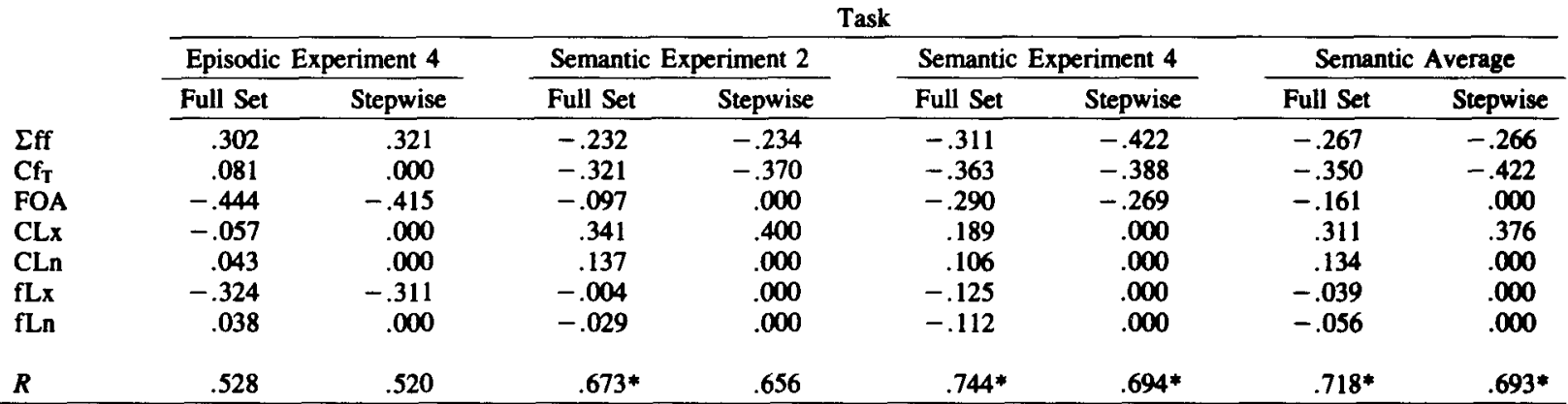

Note-NOA, number of attributes; FOA, frequency of association; CLx, lexical verification time to concept words; CLn concept length; fLx, lexical verification time to feature words; fLn, feature length; $\Sigma \mathrm{ff}$, total of average ratings of connection strength between pairs of features; $\mathrm{Cf}_{\mathrm{T}}$, rating of connection strength between concepts and features used in Experiments $2-4 . \quad d f=6,41 . \quad{ }^{*} p<.005$.

equations predicting semantic tasks. The FOA component, on the other hand, is the major predictor of performance in the episodic memory task, but it is a weak predictor of performance on the semantic tasks. As in the other regressions, the $\Sigma \mathrm{ff}$ component has a positive beta weight when predicting episodic performance and a negative beta weight when predicting performance in the semantic tasks.

\section{GENERAL DISCUSSION}

Complexity models of semantic memory predict that the time required to verify a feature of a concept should be directly related to the number of features associated with that concept; the more complex the concept, the more the time that should be required for verifying any particular feature of that concept. On the other hand, the connectivity model proposed by Klimesch (1987) predicts that, for interconnected semantic relationships, the time required to verify a feature of a concept should be inversely related to the number of features associated with that concept; the more complex the concept, the faster the verification should be . The evidence from the present experiments clearly favors the latter. In fact, not only was VT inversely related to the complexity of concept, the concept's complexity appeared to be a better predictor of VT than was even the strength of association between the concept and the feature. ${ }^{6}$

As discussed in the introduction, Klimesch (1987) found that concept complexity was also inversely related to the time required to verify that a concept was a member of a superordinate category. It should be emphasized, however, that these inverse relationships are expected only where the informational units form an interconnected structure. Specifically, an inverse relationship is not expected if the concept has a simpler (e.g., fan-like) structure. In fact, if a slightly more complex version of the 
connectivity model is considered, not only is an inverse relationship expected for interconnected structures, but a direct relationship is predicted for concepts with simple structures (See Appendix B).

In reference to Tulving's (e.g., 1983, 1985) distinction between episodic and semantic memory, one might expect that structures involving primarily semantic memory would be more likely to be interconnected than structures involving primarily memories of particular episodes; and, indeed, this seems to be the case. Klimesch (1981) obtained norms on the number of semantic and episodic features that tended to be associated with a series of concepts (presented as pictures or as words written in German to a sample of Austrian students). A second sample of students performed a semantic classification task involving the same materials. Klimesch found not only the expected inverse relationship between VTs and the number of semantic features, but also the expected direct relationship between VTs and the number of episodic features associated with the concept being classified. Other researchers have also found that the fanning effect is more likely to occur in tasks that seem to draw on episodic memory than those which seem to draw more on semantic memory (e.g., Shoben, Wescourt, \& Smith, 1978).

One application of the complexity model that has received a great deal of attention is the speed of recognition of recently studied facts. In this type of task, Anderson (e.g., 1983) has frequently found that a subject's reaction time increases as the number of facts learned about a concept increases. This increase in reaction time is " referred to as the fan effect because more facts increase the fan of propositional associations leading from a concept"' (p. 272). More recently, Anderson and Reder (1987) have found this typical fan effect if a task requires a subject to retrieve a specific fact, but they have found it to be reversed if a task requires a sensibility judgment. That is, the more facts that a subject learns about a concept, the more the time that is required to remember whether or not any particular fact is one that has been just studied, but the less the time that is required to judge whether or not a particular fact is consistent with what is known about the concept.

Anderson and Reder (1987) explain this reversible fan effect by saying that the effect of fan (i.e., in their experiments, the number of other studied sentences sharing the subject term or predicate term) is a function of the cognitive process performed.

The recognition process, which involves retrieval from long-term memory, is interfered with by the study of additional sentences. The sensibility judgment process is facilitated by the study of additional sentences. ... [This is explained] in terms of practice during study in retrieving the category of the subject and the predicate in order to compare them. (p. 365)

Although this explanation covers Anderson and Reder's (1987) differential fan effects across different cognitive tasks with the same verbal materials, it does not explain why differential fan effects are obtained when subjects perform the same cognitive task on different patterns of learned material. For example, the subjects in the Myers et al. (1984) experiments all performed a recognition memory task, but the material that was learned differed in its degree of integration. Only the low-integration condition resulted in the typical fan effect; the high-integration condition resulted in a pronounced reversed fan effect.

Thus, the type of fan effect that one obtains is a function of both the type of cognitive process being performed and the degree of integration existing with the information in the "fan." Although the informational structures developed as a result of studying sentences are undoubtedly different from those involved with existing concepts, it seems likely that the underlying processes may be similar. This explanation is most straightforward for the Myers et al. (1984) experiments, in which the degree of integration of the material can be viewed as a rough measure of the degree of interconnectivity among the facts being studied.

The connectivity hypothesis may also contribute to an explanation of the Anderson and Reder (1987) pattern of results. First, the material learned was of the lowintegration type, so that the studied facts would not, by themselves, form an interconnected structure. Thus, a slowing effect of fan would be expected, because the memory task requires that the subject differentiate the potential facts individually on the basis of what has been recently learned-a task in which the subject must rely much more on episodic than on semantic memory. Second, in the sensibility judgment task, the "practice during study in retrieving the category of the subject and the predicate" may have an effect of widening the locus of activation on the semantic components of the newly forming episodic memories. Indeed, this may be one way in which new semantic structures are developed from a series of episodic memories. If this is the case, the sensibility judgment task allows one to respond on the basis of the total activation, direct and indirect, that connects the subject and predicate on a particular test trial.

In other words, repeated exposure to the subject and/or predicate components in different contexts may well lead to a more interconnected structure. If the nature of the materials (e.g., Myers et al., 1984) or the nature of the task (e.g., the sensibility judgment task of Anderson \& Reder, 1987) allows one to make use of any source of activation, then the connectivity hypothesis predicts a reversed fan effect. If the task requires one to discriminate among the sources of activation on the basis of how the paths have been formed (e.g., the recognition memory task of Anderson \& Reder), then the connectivity hypothesis is no longer relevant, and fan would be expected to have a slowing effect on performance. This, then, may be seen as one of the key differences between the semantic and episodic tasks used in the present experiments. In both, use was made of materials that already had, prior to the experiment, an interconnected structure. However, only the semantic task allowed the subject full use of this structure for reaching the correct decision. The semantic task only required the subject to find that the concept and feature 
were related. The episodic task required that the subject discriminate which of several possible relationships had been judged within the confines of the experimental task, so that the information in semantic memory now provided distracting possibilities, rather than indirect activation. (See also Nelson et al., 1987.)

The subjects performing the semantic task in the present experiments did not study "additional" relationships for high NOA pairs within the confines of the experimental situation. In fact, the semantic congruency judgments in the present experiment (which resulted in a reaction time pattern analogous to those resulting from the sensibility judgments in Anderson \& Reder, 1987) were made by subjects who had not received any prior exposure to these words within the experiment. Thus, it would seem that, although the connectivity model explains the Anderson and Reder experiments, the Anderson and Reder model cannot explain the present results.

Whether or not this extension of the connectivity hypothesis proves useful for explaining the underlying processes of learning new facts, it seems, on the basis of both previous (e.g., Klimesch, 1981, 1987) and present experiments, to be a necessary component of models of semantic memory.

\section{REFERENCES}

Anderson, J. R. (1981). Effects of prior knowledge on memory for new information. Memory \& Cognition, 9, 237-246.

ANDERSON, J. R. (1983). A spreading activation theory of memory. Joumal of Verbal Leaming \& Verbal Behavior, 22, 261-295.

ANDERSON, J. R., REDER, L. M. (1987). Effects of number of facts studied on recognition versus sensibility judgments. Jourmal of Experimental Psychology: Leaming, Memory, \& Cognition, 13, 355-367.

Barsalou, L. W. (1982). Context-independent and context-dependent information in concepts. Memory \& Cognition, 10, 82-93.

Chang, T. (1986). Semantic memory: Facts and models. Psychological Bulletin, 99, 199-220.

Collins, A. M., LofTUS, E. F. (1975). A spreading-activation theory of semantic processing. Psychological Review, 82, 407-428.

Coluns, A. M., Q Quiluian, M.R. (1970). Does category size effect categorization time? Journal of Verbal Learning \& Verbal Behavior, 9, 432-438.

Dosher, B. A., Rosedale, G. (1991). Judgments of semantic and episodic relatedness: Common time-course and failure of segregation. Joumal of Memory \& Language, 30, 125-160.

Freedman, J. L., LofTus, E. F. (1971). Retrieval of words from long-term memory. Journal of Verbal Learning \& Verbal Behavior, 10, $107-115$.

GENTNER, D. (1981). Verb semantic structures in memory for sentences: Evidence for componential representation. Cognitive Psychology, 13, 56-83.

KINTSCH, W. (1974). The representation of meaning in memory. Hillsdale, NJ: Erlbaum.

KINTSCH, W. (1980). Semantic memory: A tutorial. In R. S. Nickerson (Ed.), Attention and performance (pp. 595-620). Hillsdale, NJ: Erlbaum.

KLIMESCH, W. (1981). Die Encodierung von Begriffen auf der Basis von Merkmalsstrukturen. Zeitschrifi fur experimentelle und angewandte Psychologie, 28, 609-636.

KuIMESCH, W. (1987). A connectivity model for semantic processing. Psychological Research, 49, 53-61.

KLIMESCH, W. (1988). Struktur und Aktivierung des Gedachtnisses. Bern: Hans Huber.

KLIMESCH, W. (in press). The structure of LTM: A connectivity model for semantic processing.
MCCLoskey, M. (1980). The stimulus familiarity problem in semantic memory research. Joumal of Verbal Learning \& Verbal Behavior, 19, 485-502.

MCCloskey, M., GlucksBerg, S. (1979). Decision processes in verifying category membership statements: Implications for models of semantic memory. Cognitive Psychology, 11, 1-37.

McNamara, T. H. P., Sternberg, R. J. (1983). Mental models of word meaning. Joumal of Verbal Learning \& Verbal Behavior, 22, 449-474.

Myers, J. L., O'Brien, E. J., BAlota, D. A., ToyofukU, M. L. (1984). Memory search without interference: The role of integration. Cognitive Psychology, 16, 217-242.

Nelson, D. L., Canas, J., BaJo, M.-T. (1987). The effects of natural category size on memory for episodic encodings. Memory \& Cognition, 15, 133-140.

RATCUFF, R., McKoon, G. (1981). Does activation really spread? Psychological Review, 88, 454-462.

ReDER, L. M., Ross, B. H. (1983). Integrated knowledge in different tasks: The role of retrieval strategy on fan effects. Journal of $E_{x}$ perimental Psychology: Learning, Memory, \& Cognition, 9, 55-72.

Rosch, E. (1975). Cognitive representations of semantic category. Journal of Experimental Psychology: General, 104, 192-233.

Shoben, E. J., Wescourt, K. T., \&Mith, E. E. (1978). Sentence verification, sentence recognition, and the semantic-episodic distinction. Journal of Experimental Psychology: Human Leaming \& Memory, 4, 304-317.

Smith, E. E., Mrdin, D. L. (1981). Categories and concepts. Cambridge, MA: Harvard University Press.

Smith, E. E., Shoben, E. J., Rips, L. J. (1974). Structure and process in semantic memory: A featural model of semantic decisions. Psychological Review, 81, 214-241.

ToGLIA, M. P., BATTIG, W. F. (1978). Handbook of semantic word norms. Hillsdale, NJ: Erlbaum.

TulviNG, E. (1983). Elements of episodic memory. New York: Oxford University Press.

Tulving, E. (1985). How many memory systems are there? American Psychologist, 40, 385-398.

\section{NOTES}

1. The details of this assumption (that the amount of activation leaving a node on any given pathway is relatively unaffected by the number of pathways connected to that node) are discussed in Appendix B.

2 . In some sense, any concept can be thought of as having an almost infinite number of fearures. What is referred to here, and what is assumed to be measured by our scales, are the attributes, features, and properties that are considered most typical and/or most necessary for a reasonable definition of the concept. They are most probably what Barsalou (1982) has referred to as a concept's context-independent information.

3. The reasons why a reversed fan effect is also expected from the feature-concept order of presentation will be discussed in more detail in the Results section of Experiment 2.

4. It should be emphasized that there is, in fact, some context in the present experiments. The superordinate category is stipulated, and the name of the category is present while the subject is making judgments about the features. By "relatively context-free," we simply mean that there is no specific context, such as would typically occur within a sentence, that might tend to emphasize some aspects of the concept and de-emphasize others. On the other hand, the stipulation of the superordinate category and the concept-as well as the restriction that the judgments are concerning features-is likely to make the norms obtained in this study, as well as in the earlier Klimesch $(1981,1987)$ studies, quite different from those that would be obtained in more general free association experiments. The purpose was to have the same amount of context information in both the normative and the judgment experiments.

5. The word pairs used in these experiments had originally been chosen with only the second type of analysis in mind. Note that there are two major differences between the two types of analyses applied to the data of this series of experiments. The obvious difference is the choice of random variable: word pairs versus subjects. The second difference is that the word pair analysis assigns a word pair to the $2 \times 2$ (NOA $x$ 
FOA) classification, regardless of its superordinate category membership, whereas the subject analysis divides the word pairs within each superordinate category into low and high NOA sets. In Appendix C, the experimental congruent pairs are preceded by a notation that indicates their respective cells in the $2 \times 2$ word pair analysis-for example, 11 indicates Lo-NOA, Lo-FOA. For the subject analysis, all eight pairs of each superordinate were divided into two equal groups on the basis of their NOA scores. Despite these differences in choice of random variable and in assignment of stimuli to independent variable classes, the two types of analyses give patterns of results that are consistent with each other and with the correlational analyses.

6. However, it also needs to be emphasized that in these experiments, the number of connections among the features of a concept was not manipulated independently of the number of features belonging to a concept. Indeed, our argument is that, when one is working with existing semantic concepts, this would be extremely difficult, if not impossible. Unfortunately, this means that there is a basic confounding within this experiment. That is, the negative fan effect found could be due directly to the number of features, rather than to the number of interconnec tions. Proponents of this argument, however, would need to explain why the use of episodic and artificial concepts leads to a positive fan effect.

\section{APPENDIX A}

In order to understand a more quantitative version of the model, consider first a completely interconnected network in which all of the possible pairs of $n$ nodes are linked. In this case, there will be the maximum number of connections: $n(n-1) / 2$. Figure Al shows such a completely interconnected network with four semantically related features and one concept node, which serves as the access node to the meaning of the concept.

Assume that activation starts from the concept node $x$ and that $\alpha$ amount of activation is received by each of the remaining $n-1$ nodes in the first step of activation via direct connections (the solid lines in Figure A1). The model does not assume reverberating activation (where an activated node immediately reflects back some fraction of its activation to the node from which it was received; cf. Anderson, 1983, p. 266). Thus, the second step consists of activation flowing from each of the $n-1$ nodes to each of the other $n-2$ nodes (the dashed lines in Figure A1). Let $\beta$ be the amount of activation sent from each of the feature nodes to each of the remaining $n-2$ feature nodes in this sec-

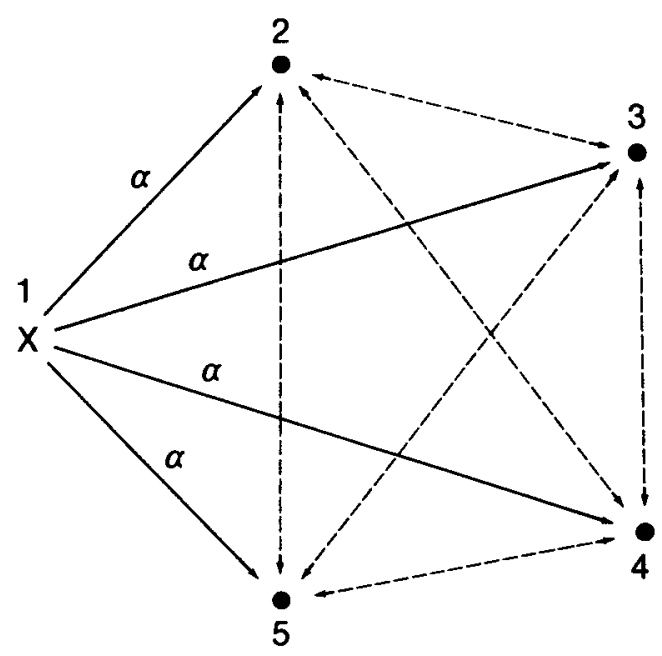

Figure A1. The first two steps of activation in a completely interconnected network of five nodes. Step 1, solid lines; Step 2, dashed lines. Not shown is the third step, in which all of the $n-1$ nodes spread their total activation back to concept nodex. ond step. Since any connection can be activated in either direction, each of the $n-1$ nodes receives an activation equal to $\beta(n-2)$. Assuming that the nodes are still active with an amount equal to $\alpha$, the total amount of activation at each node at the end of the second step would be $\alpha+\beta(n-2)$. In the third and final step, all of the $n-1$ nodes spread their total activation back to node $x$. Thus, the amount of indirect activation $I$ in any completely interconnected network with $n>2$ nodes and $n(n-1) / 2$ connections is given by

$$
I=[\alpha+\beta(n-2)](n-1) .
$$

The amount of indirect activation is thus a function of $\alpha, \beta$, and the number of nodes $n$. If, in this simplified version of the model, the values for $\alpha$ and $\beta$ are assumed to be fixed, this connectivity model predicts that the amount of indirect activation will increase as the number of nodes increases.

As with the processing assumptions underlying other network models, this model assumes that the time needed to activate a node is reciprocal to the amount of activation received by this node. Accordingly, in the first step of activation, all of the features in the network above are activated at time $1 / \alpha$. The second step of activation requires an additional time period of $1 / \beta$, and the third step lasts for $1 /[\alpha+\beta(n-2)]$ time units. Thus, the time that indirect activation requires to spread back to the concept node is equal to the sum of three terms:

$$
t(I)=1 / \alpha+1 / \beta+1 /[\alpha+\beta(n-2)] .
$$

Under the assumption that $\alpha$ and $\beta$ are fixed values, $t(\mathrm{I})$ varies only as a function of $n$, which appears only in the denominator. This means that increasing $n$ will always result in a decreasing value of $t(\mathrm{I})$, which, according to the third processing assumption, is the time needed to search a completely interconnected network.

This connectivity model is not restricted to the very particular case of the completely interconnected network. Let the average number of interconnections among the features be a fraction $f$ of the maximum number of interconnections; then $f(n-2)$ is substituted for $(n-2)$ in Formula $\mathrm{A} 1$, giving Formula $\mathrm{A} 3$, which defines the amount of indirect activation in networks that are not completely interconnected:

$$
I=[\alpha+\beta \cdot f(n-2)](n-1),
$$

where $1 \leq f(n-2) \leq(n-2)$.

Formula A3 shows that even for the minimum number of interconnections, where $f(n-2)=1$, the amount of indirect activation increases as the number of features increases.

Similarly, $f(n-2)$ must be substituted for $(n-2)$ in Formula $A 2$ in order to determine the way in which processing time is affected by the lack of a completely interconnected network:

$$
t(\mathrm{I})=1 / \alpha+1 / \beta+1 /[\alpha+\beta \cdot f(n-2)] .
$$

Again we see that $t(\mathrm{I})$ decreases as $n$ increases, so that even in the case of networks that are not completely interconnected, $t(I)$ will decrease as the number of nodes increases-as long as either $f$ remains constant for different values of $n$, or $f$ increases with increasing values of $n$. A decrease in $f$ as $n$ increases would mean that as a network gained features, it would become less interconnected. This is not expected to occur within semantic memory under the representation assumption stated earlier.

Thus, a network will be termed interconnected if all of the $n-1$ features are directly linked to the concept node and all $n-1$ features are linked to at least one of the other $n-2$ features. Given this definition and the assumptions described above, including the assumption that the average number of interconnec- 
tions, $f(n-2)$, is not an inverse function of $n$, the connectivity model predicts that concepts with many core features can be processed faster than concepts with only a few core features.

So far the discussion has been limited to the simplest possible case in which the search begins from a single node. Usually, however, the search begins from at least two nodes. Consider a semantic judgment task of the following type: Does concept $X$ (e.g., Canary) belong to superordinate category $Y$ (e.g., BIRD)? In this case, the search process starts from the two concept nodes, $X c$ and $Y c$, and a semantic decision is performed on the basis of a feature intersection process. That is, more indirect activation spreads back to $X c$ and $Y c$ on positive trials than on negative trials, because activations from different sources only meet via common features. Given that increased indirect activation speeds processing time (the third processing assumption), the important implication is that positive decisions will be reached faster than negative decisions. Furthermore, the speed of positive decisions will be affected by three separate factors: the number of features of concept $X$, the number of features of concept $Y$, and the number of features shared by concepts $X$ and $Y$.

\section{APPENDIX B \\ A Discussion of the Relationship between the Amount of Activation Leaving a Node and the Number of Outgoing Pathways from that Node}

The type of function expected between the number of features belonging to a concept and the time required to verify that the concept has a given feature is determined by the relationship that is assumed to exist between the amount of activation leaving a node on a given pathway and the number of pathways connected to that node. In the following three possibilities, $\tilde{n}$ (equal to $n-1$ ) is the number of pathways leaving a node, $A_{\mathrm{i}}$ is the amount of input activation, $\alpha$ is the amount of activation leaving a given node along any one pathway, and $A_{\mathrm{o}}$ is the total amount of output activation leaving any given node; that is, the value of $A_{0}$ for a given node is equal to the sum of all the $\alpha$ values for that node.
Possibility 1. If $A_{\mathrm{o}}=A_{\mathrm{i}}$, then $\alpha=A_{\mathrm{i}} / \tilde{n}$, and $\alpha$ always decreases as $\tilde{n}$ increases. Since the time required in the first step of the activation process is a function of $1 / \alpha$, this predicts the traditional fan effect; the greater the number of features belonging to a concept, the slower should be the verification time. This is typically found with concepts having simple structures-most notably with concepts that are artificially defined within the confines of the experiment (i.e., "simple episodic concepts").

Possibility 2. If the amount of activation leaving a node on any given pathway is unaffected by the number of pathways connected to that node, then $\alpha=A_{\mathrm{i}}$, and, when $\tilde{n}>1, A_{\mathrm{o}}>A_{\mathrm{i}}$. As explained in the text, this predicts that verification time should decrease with concept complexity in interconnected models. However, if a concept has a simple fan-like structure, this assumption predicts no change in verification time as a function of number of features, rather than the increase in verification time that is typically found.

Possibility 3. If, as in Possibility 2, $A_{0}>A_{i}$, but the value of $\alpha$ is some decreasing function of $\tilde{n}$, then the model will predict both the direct function between verification time and number of features when the concepts have simple structures, and the inverse function when the concepts have interconnected structures. For example, if $\alpha$ is equal to $A_{\mathrm{i}}(\tilde{n}+1) / \tilde{n}$, then $\alpha$ will decrease as $\tilde{n}$ increases-but in such a way that, if the informational units form an interconnected structure, the increase in activation coming from indirect activation will more than offset even much greater decreases. This increase in indirect activation, then, will in fact result in the predicted inverse time function.

We expect that the connectivity model, as it evolves, will include the assumption stated in Possibility 3 above. In addition, a more refined version will eventually need to recognize that not all pathways within a structure carry the same load of activation; i.e., that is, $\alpha_{\mathrm{i}}$ will not be uniform over all $i$. This might be represented by pathways of different length or of different width. Such refinements can be expected to greatly improve the size of the correlation between verification time and the number of features. The measure $\Sigma$ ff obtained in Experiment 1 is a first attempt of such a refinement; however, the main purpose of the present paper is to suggest the direction of a new class of semantic memory models.

\section{APPENDIX C \\ Concept-Feature Pairs used in the Present Experiments}

For the experimental congruent pairs, NOA (number of attributes), NOF (number of features), and $\Sigma \mathrm{ff}$ (sum of rated connection strength between pairs of features) values are given for the concepts. The FOA (frequency of association) and $\mathbf{C} \mathbf{f}_{\mathbf{T}}$ (rated connection strength between concept and feature tested) values are given for the pairs. In addition, the average lexical verification times are given for the concepts, CLx, and for the features, fLx.

The assignment of congnent word pairs to conditions in the analyses with word pairs as the random variable is indicated by pairs of numbers preceeding the pair:

$\begin{array}{lll}11 & \text { Lo-NOA; } & \text { Lo-FOA } \\ 12 & \text { Lo-NOA; } & \text { Hi-FOA } \\ 21 & \text { Hi-NOA; } & \text { Lo-FOA } \\ 22 & \text { Hi-NOA; } & \text { Hi-FOA }\end{array}$

Marked (*) congruent pairs were not included in these analyses.

Practice Superordinates: TOOLS and UTENSILS

\begin{tabular}{ll}
\multicolumn{2}{c}{ Congruent } \\
\hline PAMMMER & HEAVY \\
KNIFE & STEEL \\
SAW & TEETH \\
PENCIL & WRITING
\end{tabular}

\begin{tabular}{ll}
\multicolumn{2}{r}{ Incongruent Pairs } \\
\hline DRILL & CUTS \\
CHISEI & SOFT \\
AX & INK \\
SCISSORS & ELASTIC
\end{tabular}


Practice Superordinates: ANIMALS and FURNITURE

\begin{tabular}{ll} 
& CONGRUENT PAIRS \\
\hline LAMP & SHINES \\
ELEPHANT & TRUNK \\
DOG & TAIL \\
TABLE & LEGS
\end{tabular}

\begin{tabular}{ll}
\multicolumn{2}{c}{ INCONGRUENT PAIRS } \\
\hline PILLOW & HARD \\
CAT & BARKS \\
TURTLE & FAST \\
ROCKER & STABLE
\end{tabular}

Practice Superordinates: CONTAINERS and WEATHER PHENOMENA

\begin{tabular}{|c|c|c|c|}
\hline \multicolumn{2}{|c|}{ Congruent Pairs } & \multicolumn{2}{|c|}{ INCONGRUENT PAIRS } \\
\hline CAGE & BARS & BOTTLE & FUR \\
\hline BOWL & CLAY & SUN & COLD \\
\hline RAIN & WET & BOX & WINDY \\
\hline TORNADO & DESTRUCTION & SNOW & FOOD \\
\hline
\end{tabular}

Experimental Superordinate: BIRDS

\begin{tabular}{|c|c|c|c|c|c|c|c|c|c|}
\hline \multirow[b]{2}{*}{21} & \multicolumn{2}{|c|}{ Congruent Pairs } & \multirow{2}{*}{$\begin{array}{c}\text { NOA } \\
3.89\end{array}$} & \multirow{2}{*}{$\begin{array}{c}\text { NOF } \\
6\end{array}$} & \multirow{2}{*}{$\begin{array}{l}\text { Iff } \\
40.9\end{array}$} & \multirow{2}{*}{$\begin{array}{c}\text { FOA } \\
39.3\end{array}$} & \multirow{2}{*}{$\begin{array}{c}C f_{\mathrm{T}} \\
4.53\end{array}$} & \multirow{2}{*}{$\begin{array}{c}\text { CLx } \\
796\end{array}$} & \multirow{2}{*}{$\begin{array}{l}f L x \\
686\end{array}$} \\
\hline & BLUEJAY & FEATHERS & & & & & & & \\
\hline 22 & CROW & BLACK & 3.83 & 8 & 49.0 & 92.9 & 5.54 & 639 & 587 \\
\hline$*$ & DUCK & SWIMS & 4.18 & 8 & 52.6 & 39.3 & 3.67 & 571 & 703 \\
\hline$*$ & EAGLE & CLAWS & 3.87 & 10 & 106.2 & 14.3 & 3.60 & 623 & 613 \\
\hline 21 & HAWK & BEAK & 4.03 & 6 & 43.0 & 28.6 & 3.79 & 671 & 786 \\
\hline 12 & LARK & SINGS & 3.45 & 4 & 16.9 & 46.4 & 4.00 & 802 & 664 \\
\hline 11 & ROBIN & WINGS & 3.65 & 6 & 33.5 & 35.7 & 4.69 & 625 & 584 \\
\hline 11 & SWALLOW & FLIES & 3.05 & 6 & 35.6 & 35.7 & 4.47 & 710 & 669 \\
\hline
\end{tabular}

\begin{tabular}{ll}
\multicolumn{2}{c}{ Incongruent } \\
\hline Pairs \\
CANARY & BLUE \\
CHICKEN & DIVES \\
OWL & SOARS \\
PELICAN & COOING \\
PENGUIN & CHIRPS \\
PIGEON & QUACKS \\
SPARROW & PREDATORY \\
\end{tabular}

\begin{tabular}{|c|c|c|c|c|c|c|c|c|c|}
\hline \multicolumn{10}{|c|}{ Experimental Superordinate: CLOTHES } \\
\hline & \multicolumn{2}{|c|}{ Congruent Pairs } & NOA & NOF & $\Sigma \mathrm{ff}$ & FOA & $\mathrm{Cf}_{\mathbf{T}}$ & CLX & fLx \\
\hline 11 & BLOUSE & PRETTY & 3.75 & 6 & 41.0 & 21.4 & 4.08 & 732 & 664 \\
\hline * & $\operatorname{COAT}$ & SLEEVES & 3.89 & 6 & 40.7 & 25.0 & 4.62 & 577 & 752 \\
\hline 21 & JACKET & HOOD & 4.26 & 8 & 74.0 & 25.0 & 3.08 & 597 & 676 \\
\hline 11 & PAJAMA & WARM & 3.50 & 2 & 4.2 & 37.7 & 5.54 & 799 & 643 \\
\hline * & PANTS & POCKETS & 4.09 & 6 & 30.6 & 39.3 & 4.62 & 665 & 659 \\
\hline 21 & SHIRT & COLLAR & 4.23 & 7 & 55.9 & 25.0 & 4.85 & 633 & 688 \\
\hline 11 & socks & COrTON & 3.15 & 3 & 9.5 & 28.6 & 5.00 & 663 & 608 \\
\hline 12 & VEST & BUTTONS & 3.32 & 4 & 8.2 & 42.9 & 3.46 & 774 & 583 \\
\hline
\end{tabular}

\begin{tabular}{ll}
\multicolumn{2}{r}{ Incongruent } \\
\hline POAirs \\
CAPE & FRILL \\
DRESS & HEELS \\
HAT & SOLE \\
SANDAL & BELT \\
SHOES & ZIPPERS \\
SKIRT & ARM \\
STOCKING & TOES \\
& EAR
\end{tabular}

Experimental Superordinate: FRUITS

\begin{tabular}{|c|c|c|c|c|c|c|c|c|c|}
\hline \multirow[b]{2}{*}{22} & \multicolumn{2}{|c|}{ Congruent Pairs } & \multirow{2}{*}{$\begin{array}{c}\text { NOA } \\
3.57\end{array}$} & \multirow{2}{*}{$\begin{array}{c}\text { NOF } \\
9\end{array}$} & \multirow{2}{*}{$\begin{array}{l}\Sigma \text { ff } \\
90.7\end{array}$} & \multirow{2}{*}{$\begin{array}{c}\text { FOA } \\
96.4\end{array}$} & \multirow{2}{*}{$\begin{array}{c}\mathrm{Cf}_{\mathrm{T}} \\
4.13\end{array}$} & \multirow{2}{*}{$\begin{array}{c}\text { CLx } \\
532\end{array}$} & \multirow{2}{*}{$\begin{array}{l}\text { fLx } \\
610\end{array}$} \\
\hline & APPLE & RED & & & & & & & \\
\hline 12 & BANANA & YELLOW & 3.28 & 2 & 2.1 & 89.3 & 5.40 & 688 & 573 \\
\hline$*$ & BERRY & SMALL & 3.77 & 7 & 53.1 & 35.7 & 4.80 & 685 & 609 \\
\hline * & CHERRY & PIT & 3.49 & 7 & 46.8 & 57.1 & 4.60 & 572 & 630 \\
\hline
\end{tabular}




$\begin{array}{lllllrllll}11 & \text { LEMON } & \text { BITTER } & 3.63 & 4 & 13.6 & 21.4 & 3.93 & \mathbf{6 2 4} & \mathbf{6 6 0} \\ 22 & \text { PEACH } & \text { FUZZY } & 3.59 & 7 & 53.7 & \mathbf{6 0 . 7} & \mathbf{5 . 4 0} & \mathbf{6 2 3} & \mathbf{6 6 1} \\ 11 & \text { PEAR } & \text { SWEET } & 3.02 & 3 & 7.2 & 39.3 & 4.60 & \mathbf{5 6 6} & \mathbf{5 9 3} \\ 12 & \text { PLUM } & \text { PURPLE } & 3.18 & 6 & 36.9 & \mathbf{7 8 . 6} & \mathbf{5 . 1 3} & \mathbf{6 0 8} & \mathbf{6 8 9}\end{array}$

\begin{tabular}{ll}
\multicolumn{2}{c}{ Incongruent Pairs } \\
\hline GRAPE & STINGS \\
LIME & THORNY \\
OLIVE & CREEPS \\
ORANGE & FLAT \\
PINEAPPLE & TINY \\
RASPBERRY & HOT \\
STRAWBERRY & SALINE \\
TANGERINE & VIOLET
\end{tabular}

Experimental Superordinate: VEGETABLES

\begin{tabular}{|c|c|c|c|c|c|c|c|}
\hline \multicolumn{2}{|c|}{ Congruent Pairs } & NOA & NOF & $\Sigma f f$ & FOA & $C f_{\mathrm{T}}$ & CLx \\
\hline CARROT & LONG & 3.25 & 3 & 4.3 & 60.7 & 3.53 & 668 \\
\hline CORN & $\mathrm{COB}$ & 4.10 & 6 & 33.6 & 50.0 & 5.00 & 589 \\
\hline CUCUMBER & SEEDS & 3.18 & 3 & 4.0 & 53.6 & 2.80 & 715 \\
\hline GARLIC & WHITE & 2.77 & 2 & 1.0 & 50.0 & 3.93 & 706 \\
\hline LETTUCE & GREEN & 3.44 & 5 & 26.9 & 85.7 & 5.07 & 639 \\
\hline ONION & ROUND & 3.25 & 5 & 15.6 & 50.0 & 4.00 & 615 \\
\hline SPINACH & LEAF & 3.14 & 3 & 9.0 & 42.9 & 4.40 & 676 \\
\hline TOMATO & JUICY & 3.48 & 4 & 10.7 & 39.3 & 3.87 & 618 \\
\hline
\end{tabular}

\begin{tabular}{ll}
\multicolumn{2}{c}{ Incongruent } \\
\hline Pairs \\
\hline BEET & GREY \\
BEAN & HEAD \\
CAULIFLOWER & POINTED \\
CABBAGE & BROWN \\
PEA & HUGE \\
POTATO & SOUR \\
RICE & SHARP \\
SPROUT & POD
\end{tabular}

Experimental Superordinate: VEHICLES

\begin{tabular}{|c|c|c|c|c|c|c|c|c|c|}
\hline & \multicolumn{2}{|c|}{ Congruent Pairs } & NOA & NOF & $\Sigma$ ff & FOA & $\mathrm{Cf}_{\mathbf{T}}$ & CLx & fLx \\
\hline 22 & CAR & WHEELS & 5.74 & 7 & 45.0 & 60.7 & 4.64 & 598 & 627 \\
\hline 21 & JEEP & TIRES & 4.47 & 6 & 48.7 & 17.9 & 4.64 & 654 & 683 \\
\hline 21 & JET & PILOT & 4.89 & 6 & 42.2 & 35.7 & 5.07 & 616 & 584 \\
\hline$*$ & SCOOTER & MOTOR & 3.89 & 4 & 12.3 & 32.1 & 4.50 & 745 & 667 \\
\hline 21 & TRACTOR & PLOWS & 4.30 & 5 & 18.2 & 35.7 & 4.93 & 743 & 803 \\
\hline 11 & TROLLEY & TRACKS & 3.70 & 5 & 23.1 & 25.0 & 5.21 & 940 & 717 \\
\hline 21 & TRUCK & ENGINE & 4.84 & 8 & 63.8 & 32.1 & 4.71 & 617 & 771 \\
\hline & YACHT & SAILS & 4.22 & 5 & 36.2 & 42.9 & 4.36 & 751 & \\
\hline
\end{tabular}

\begin{tabular}{|c|c|}
\hline \multicolumn{2}{|c|}{ Incongruent Pairs } \\
\hline BICYCLE & WINDOWS \\
\hline BOAT & BUMPER \\
\hline Bus & BELL \\
\hline CANOE & CLUTCH \\
\hline LOCOMOTIVE & DECK \\
\hline SHIP & CABOOSE \\
\hline TRAILER & FLOATS \\
\hline TRAJN & FISHING \\
\hline
\end{tabular}

Experimental Superordinate: WEAPONS

\begin{tabular}{|c|c|c|c|c|c|c|c|c|c|}
\hline \multirow[b]{2}{*}{22} & \multicolumn{2}{|c|}{ Congruent Pairs } & \multirow{2}{*}{$\begin{array}{c}\text { NOA } \\
4.52\end{array}$} & \multirow{2}{*}{$\begin{array}{c}\text { NOF } \\
7\end{array}$} & \multirow{2}{*}{$\begin{array}{l}\text { Eff } \\
95.4\end{array}$} & \multirow{2}{*}{$\begin{array}{c}\text { FOA } \\
53.6\end{array}$} & \multirow{2}{*}{$\begin{array}{c}\mathbf{C f}_{\mathrm{T}} \\
5.50\end{array}$} & \multirow{2}{*}{$\begin{array}{c}\text { CLx } \\
658\end{array}$} & \multirow{2}{*}{$\begin{array}{l}\mathrm{fLx} \\
742\end{array}$} \\
\hline & ВОМв & EXPLODES & & & & & & & \\
\hline 22 & Bow & STRING & 3.87 & 4 & 15.5 & 42.9 & 4.43 & 778 & 613 \\
\hline 22 & CANNON & BALLS & 3.92 & 5 & 18.3 & 60.7 & 4.79 & 716 & 648 \\
\hline$*$ & CLUB & WOODEN & 4.03 & 2 & 1.6 & 39.3 & 4.57 & 633 & 725 \\
\hline
\end{tabular}


KROLL AND KLIMESCH

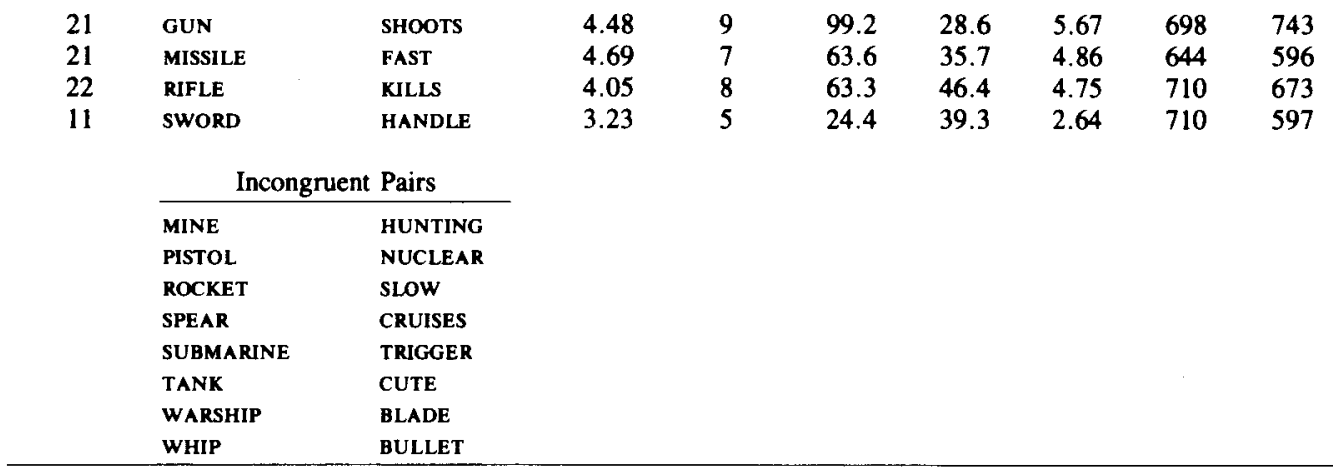

(Manuscript received May 19, 1989;

revision accepted for publication August 30, 1991.) 